\title{
Tungsten geochemistry and implications for understanding the Earth's interior
}

\author{
Ricardo Arevalo Jr. *, William F. McDonough \\ Department of Geology, University of Maryland, College Park, MD 20742, USA
}

\section{A R T I C L E I N F O}

\section{Article history:}

Received 25 February 2008

Received in revised form 21 May 2008

Accepted 22 May 2008

Available online 7 June 2008

Editor: R.W. Carlson

\section{Keywords:}

tungsten

uranium

basalt

core

mantle

concentration ratio

\begin{abstract}
A B S T R A C T
The concentration of tungsten (W) in basaltic melts provides a window into the behavior of this element during core-mantle separation, crust formation, silicate differentiation, and potentially core-mantle interaction. We have analyzed an extensive suite of modern basalts $(n=86)$ for their trace element chemistry via laser ablation ICP-MS, with barium (Ba), thorium (Th), uranium (U), and W concentrations typically determined to $\leq 5 \%(2 \sigma)$ uncertainty. We find that the partitioning behavior of $\mathrm{U}$ mirrors that of $\mathrm{W}$ during basalt genesis, whereas Ba and Th both behave more incompatibly. The $\mathrm{W} / \mathrm{U}$ ratio of our complete sample suite $(0.65 \pm 0.45,2 \sigma)$ is representative of the mean modern mantle, and is indistinguishable from that of mid-ocean ridge basalts ( $\left.\mathrm{W} / \mathrm{U}_{\mathrm{MORB}}=0.65 \pm 0.41, n=52\right)$, ocean island basalts $\left(\mathrm{W} / \mathrm{U}_{\mathrm{OIB}}=0.63 \pm 0.07, n=10\right)$, and back-arc basin basalts $\left(\mathrm{W} / \mathrm{U}_{\mathrm{BABB}}=0.62 \pm 0.09, n=12\right)$. This ratio is also consistent with the $\mathrm{W} / \mathrm{U}$ ratio of the continental crust, and thus represents the W/U ratio of the entire silicate portion of the Earth.

Assuming a concentration of $20 \pm 8(2 \sigma) \mathrm{ng} / \mathrm{g} \mathrm{U}$ in the bulk silicate Earth, the abundance of W in the silicate Earth is $13 \pm 10 \mathrm{ng} / \mathrm{g}$. Following mass balance, this implies a mean modern mantle and core composition of $8.3 \pm 7.1 \mathrm{ng} / \mathrm{g} \mathrm{W}$ and $500 \pm 120 \mathrm{ng} / \mathrm{g} \mathrm{W}$, respectively. Additionally, the MORB source is modeled to contain approximately $3.0 \pm 2.3 \mathrm{ng} / \mathrm{g} \mathrm{W}$, indicating a four-fold depletion of the highly incompatible elements in the MORB source relative to the silicate Earth.

Although both the isotopic composition of $\mathrm{W}$ and the constancy of the silicate Earth $\mathrm{W} / \mathrm{U}$ ratio allow for potential insight into core-mantle exchange, both of these proxies are extremely dependent on the chemical composition of the source. A case study of three Hawaiian picrites with enrichments in ${ }^{186}$ Os- ${ }^{187}$ Os but terrestrial $\varepsilon_{182 \mathrm{w}}$ can be explained by: i) a lack of a core component in the Hawaiian "plume," ii) crustal contamination, or iii) a source composition enriched in incompatible trace elements relative to the bulk silicate Earth.
\end{abstract}

Published by Elsevier B.V.

\section{Introduction}

The metallic core separated from the silicate portion of the Earth some 10 to 70 million years after the formation of the solar system (e.g., Kleine et al., 2004a; Jacobsen, 2005), during which $>90 \%$ of the planet's budget of tungsten (W) was sequestered into the core (Jagoutz et al., 1979; Sun, 1982; Newsom and Palme, 1984). The distribution of $\mathrm{W}$, along with many other siderophile (iron-loving) elements, has been established by chemical differentiation processes that were controlled by a spectrum of pressure, temperature and oxygen fugacity conditions as the Earth has evolved (e.g., Righter, 2003 and references therein). Following core formation, however, the geochemical behavior of $\mathrm{W}$ in the silicate Earth has been dominantly lithophilic (silicate-loving) and highly incompatible during mantle melting and crust formation (Newsom et al., 1996 and references therein); therefore, $\mathrm{W}$ provides insights into both core-mantle segregation and the differentiation of the silicate Earth.

\footnotetext{
* Corresponding author. Tel.: +1 301405 6248; fax: +1 3014053597.

E-mail addresses: rarevalojr@gmail.com, gatorick@umd.edu (R. Arevalo).
}

Recently, the isotopic composition of $\mathrm{W}$ has been used as a shortterm geochronometer for dating planetary and asteroidal core formation (e.g., Kleine et al., 2002; Schoenberg et al., 2002; Yin et al., 2002; Kleine et al., 2004a, 2005a) and early silicate differentiation (e.g., Righter and Shearer, 2003; Kleine et al., 2004b, 2005b). Tungsten isotopic anomalies have also been implicated as a potentially robust geochemical tracer for core-mantle interactions (Scherstén et al., 2004; Brandon and Walker, 2005). However, the geochemical behavior of $\mathrm{W}$ in the silicate portion of the Earth is not wellconstrained, largely due to its highly incompatible behavior during mantle melting and the depleted composition of mantle-derived basalts. Estimates of the abundance of $\mathrm{W}$ in the silicate Earth range from as low as $7 \mathrm{ng} / \mathrm{g}$ to as high as $29 \mathrm{ng} / \mathrm{g}$ (Jagoutz et al., 1979; Sun, 1982; Sims et al., 1990; McDonough and Sun, 1995; Newsom et al., 1996; Palme and O'Neill, 2003; Lyubetskaya and Korenaga, 2007). Defining the abundance and distribution of $\mathrm{W}$ within the Earth is imperative to: i) understand its geochemical behavior, ii) assess the usefulness of $\mathrm{W}$ as a tracer of geochemical processes, iii) quantitatively model the composition of the MORB (mid-ocean ridge basalt) source and any potential deep mantle reservoir, and iv) investigate putative core-mantle interactions through both $\mathrm{W}$ isotopes and concentration 
ratios. Here we report high-precision concentration data for $\mathrm{Ba}, \mathrm{Th}, \mathrm{U}$, and $\mathrm{W}$ in an extensive suite of oceanic basalts in order to define the abundance of $\mathrm{W}$ in the modern mantle, bulk silicate Earth and core.

\section{The abundance of $W$ in the silicate Earth}

\subsection{The geochemical behavior of $W$}

As a refractory element, the initial abundance of $\mathrm{W}$ in the bulk Earth can be calculated from chondritic relative abundances without a volatility correction. Estimates of $\mathrm{W}$ in the silicate portion of the Earth, however, are poorly constrained. Difficulties in determining the abundance of $\mathrm{W}$ in the silicate Earth stem from the binary behavior of the element; whereas W acts as a moderately siderophile element under reducing conditions (i.e., during core formation), it has behaved as an extremely incompatible lithophile element during the differentiation of the silicate Earth (Palme and Rammensee, 1981). Consequently, $\mathrm{W}$ is strongly enriched in the core and continental crust, respectively, leaving the modern mantle depleted.

In the silicate Earth, the abundances of siderophile elements that are compatible during mantle melting $\left(D^{\text {sil-crystal/sil-liq }}>1\right.$; e.g., Ni and Co) are established from studies of massif peridotites and mantle xenoliths because these elements are largely retained in olivine during partial melting. However, in order to determine the silicate Earth abundances of siderophile elements that behave incompatibly during mantle melting, it is necessary to determine the abundances of these elements in both the modern mantle and continental crust. Knowledge of the composition and relative mass contributions of the mantle and crustal reservoirs allows a reconstruction of the concentration of these elements in the silicate Earth to be calculated (Newsom, 1990; Sims et al., 1990).

\subsection{Incompatible element concentration ratios}

The concentration of incompatible elements in basaltic melts is controlled by the concentration of these elements in the source mantle as well as a variety of fractionation processes, including partial melting, fractional crystallization and crustal contamination. Concentration ratios of similarly incompatible trace elements provide a reliable estimate of the trace element composition of the source and can complement information derived from radiogenic isotopes (Hofmann, 2003 and references therein). Concentration ratios must be used with care, however, because unlike isotope ratios they may be fractionated during processes of magma genesis. A concentration ratio that is uniform in all types of oceanic basalts (e.g., mid-ocean ridge basalts, MORB, oceanic island basalts, OIB, and back-arc basin basalts, BABB) and is independent of the absolute concentrations of the elements involved represents a mantle value that has been unfractionated by silicate differentiation. Such constant ratios (e.g., $\mathrm{Zr} / \mathrm{Hf}, \mathrm{Sm} / \mathrm{Hf}, \mathrm{Rb} / \mathrm{Ba}$, $\mathrm{Nb} / \mathrm{Ta}$, etc.) reflect element pairs with nearly identical bulk partition coefficients during the genesis of basaltic partial melts and may be inferred to be representative of their source ratios (Hofmann et al., 1986; Newsom et al., 1986; Sun and McDonough, 1989). Constant concentration ratios of highly incompatible elements are particularly likely to represent the trace element ratios of their source regions, as these elements are quantitatively removed from their source after only a few percent of partial melting (Salters and Stracke 2004).

Because $\mathrm{W}$ is one of the most incompatible elements during mantle melting, a concentration ratio involving $\mathrm{W}$ and another similarly incompatible element (e.g., W/Ba, W/Th or W/U) likely characterizes the ratio of the modern mantle if the ratio remains constant throughout a range of MORB, OIB and BABB samples. Although no other element behaves exactly like $\mathrm{W}$ in all geologic processes, previous work suggests that the geochemical behavior of $\mathrm{W}$ in silicate systems is most comparable to that of $\mathrm{Ba}$, Th and $\mathrm{U}$ (Newsom and Palme, 1984; Newsom et al., 1986, Sims et al., 1990; Newsom et al., 1996; Noll et al., 1996).

\subsection{Tungsten in the bulk continental crust}

Newsom et al. (1996) estimated the abundance of $\mathrm{W}$ in the silicate Earth by measuring relatively constant $\mathrm{W} / \mathrm{Th}$ ratios in representative samples of both the continental crust and modern mantle. In order to characterize the abundance of $\mathrm{W}$ in the bulk crust, they examined a comprehensive collection of samples representative of the lower, middle and upper crustal reservoirs; over 145 crustal samples were analyzed, including 18 lower crustal xenoliths, 20 rocks from highgrade metamorphic terrains, 45 samples of continental sediments, 13 rocks from oceanic volcanic arcs, 35 rocks from continental volcanic arcs, 8 continental basalts, and 7 komatiites. Their study, which established the concentration of W in the continental crust as $\sim 1000 \mathrm{ng} / \mathrm{g}$, still serves as the standard for examining the abundance of $\mathrm{W}$ in the bulk continental crust (Rudnick and Gao, 2003).

\subsection{Tungsten in the modern mantle}

Newsom et al. (1996) also estimated the abundance of $W$ in the modern mantle, but they analyzed only a limited sample set of mantle-derived oceanic basalts $(n=20)$ and altered mantle nodules

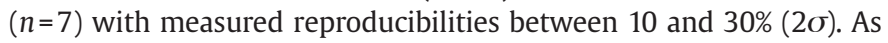
opposed to mantle xenoliths, examining a broad spectrum of MORB, OIB and BABB is instrumental to constraining the abundance of $\mathrm{W}$ in different mantle reservoirs because oceanic basalts represent the most significant volume of terrestrial magmatism (an average rate of $\sim 30 \mathrm{~km}^{3} / \mathrm{yr}$ of basalt has been produced over the past $180 \mathrm{Myr}$; Crisp, 1984) and carry the smallest risk of being contaminated during magma transport (Jochum et al., 1989; Hofmann, 1997). In this study, 86 mantle-derived samples are analyzed, including 52 MORB, 16 intraplate basalts and 18 convergent margin samples. The reproducibility of our measurements (typically $\leq 5 \%, 2 \sigma$ ) are improved over previous studies, and the extensive compilation of mantle-derived materials considered here characterizes source regions from various depths of the mantle, a wide spatial distribution across the globe and every major tectonic setting.

\section{Materials and methods}

\subsection{Sample descriptions}

The mantle rocks analyzed in this study include both normal-type MORB (N-MORB, K/Ti $\leq 0.140$ ) and enriched-type MORB (E-MORB, K/ $\mathrm{Ti}>0.140$ ), as well as a multitude of intraplate basalts, island arc volcanics, and back-arc basin basalts. Most of the rocks are oceanic basaltic glasses or fine-grained scoria, ranging from 40 to $59 \mathrm{wt} . \% \mathrm{SiO}_{2}$ and 3 to $10 \mathrm{wt} . \% \mathrm{MgO}$; thus, a range of magma compositions, from primitive to differentiated, are represented by this sample set. More information regarding the diversity of these samples, including a map showing their global distribution, is provided in the accompanying Supplementary materials.

\subsection{Analytical method}

Compared to rocks with coarser textures, glassy and/or finegrained samples provide comparatively homogeneous surface compositions that can be analyzed via laser ablation mass spectrometry. This in-situ method of measuring trace element concentrations produces high-precision data and has been validated through numerous analytical studies (e.g., Pearce et al., 1997; Eggins et al., 1998; Norman et al., 1998; Eggins and Shelley, 2002; Jochum et al., 2005, 2006, 2007). Laser ablation mass spectrometry also allows for: i) analyses of fresh, unaltered materials, ii) low blanks, iii) millimeter-scale sample specimens, iv) spatially resolved, ng/g-level chemical measurements, and v) surface and grain-boundary contamination to be avoided. All samples examined in this study were analyzed using a New Wave 
Table 1

LA-ICP-MS specifications

Laser ablation parameters

Ablation pattern

Scan speed

Spot size (diameter)

Repetition rate

Energy density

Mass spectrometer parameters

Forward power

HV

Scan optimization

Number of pre-scans

Active dead time

Cool gas flow

Auxiliary gas flow

Sample gas flow

Carrier (He) gas flow

Cones

${ }^{\text {a }}$ Laser parameters were programmed to produce ideal photon fluences between and $2 \mathrm{~J} / \mathrm{cm}^{2}$.

frequency-quintupled Nd-YAG laser (213 nm light) coupled to a Thermo Finnigan Element2 single-collector ICP-MS at the University of Maryland.

The laser and mass spectrometer parameters utilized for the measurements of this study are provided in Table 1. Prior to sample analysis, the mass spectrometer was tuned to maximize signal (based on ${ }^{43} \mathrm{Ca}$ and ${ }^{232} \mathrm{Th}$ spectra) and minimize oxide production $\left({ }^{232} \mathrm{Th}^{16} \mathrm{O} /{ }^{232} \mathrm{Th}<0.20 \%\right)$. The detection parameters included a dwell time of $10 \mathrm{~ms}$ for ${ }^{135,}{ }^{137} \mathrm{Ba}$, $30 \mathrm{~ms}$ for ${ }^{232} \mathrm{Th}$ and ${ }^{238} \mathrm{U}$, and $100 \mathrm{~ms}$ for ${ }^{182,} 183,184,{ }^{186} \mathrm{~W}$ in order to accumulate adequate counting statistics to evaluate and constrain isobaric interferences, thus increasing analytical precision.

Spectral matrix effects (i.e., isobaric interferences) were considered and monitored during analysis in order to prevent a compromise in accuracy. In this study, the only potential elemental isobaric interference is ${ }^{186} \mathrm{Os}$ at mass ${ }^{186} \mathrm{~W}$, but the concentration of Os in our basaltic melts was lower than our lower limit of detection. A potentially more problematic source of isobaric interferences is diatomic oxides. However, our analytical protocol measured the concentration of W by monitoring four isotope mass stations: ${ }^{182} \mathrm{~W},{ }^{183} \mathrm{~W},{ }^{184} \mathrm{~W}$, and ${ }^{186} \mathrm{~W}$. Any potential oxide interferences on these masses would yield discordant results between isotope ratios, but no systematic variances were detected (see Supplementary materials). Further, we conducted an experiment in which we analyzed a synthetic basaltic glass doped with 500 ppm of the heavy rare-earth elements (HREE). Employing the analytical parameters described above, HREE-oxide production rates (i.e., $\mathrm{YbO} / \mathrm{Yb}$ and $\mathrm{TmO} / \mathrm{Tm}$ ) were measured to be $\sim 10^{-6}$. Accordingly, HREE-oxides do not present significant isobaric interferences to even the most depleted $\mathrm{W}$ concentrations in our natural samples.

Non-spectral matrix effects in concentration measurements acquired through laser ablation ICP-MS can result from differences in chemical compositions between the analyte and the standard reference material. Although NIST SRM 612 (the standard reference material utilized for our measurements) has been well characterized and found to be homogeneous with respect to $\mathrm{Ba}, \mathrm{Th}, \mathrm{U}$, and $\mathrm{W}$, this glass represents a more enriched composition (with approximately $40 \mu \mathrm{g} / \mathrm{g}$ of each of these elements) than oceanic basalts, which can be far more depleted, particularly in regard to Th, $\mathrm{U}$ and $\mathrm{W}$. In order to account for this discrepancy, which could potentially lead to analytical biases, we have externally calibrated our $\mathrm{W}$, Th and $\mathrm{U}$ measurements to a suite of rocks analyzed via solution ICP-MS, including both isotope dilution and high-precision standard addition analyses. More details on the calibration of our trace element analyses are provided in the Supplementary materials.

\section{Results}

\subsection{Data precision and accuracy}

Concentrations and uncertainties of Ba, Th, U, and W for all 86 samples investigated in this study are reported in Table 2. Concentrations of $\mathrm{W}$ range from $<10 \mathrm{ng} / \mathrm{g}$ in the most depleted MORB to $>2000 \mathrm{ng} / \mathrm{g}$ in the most enriched intraplate basalt. Most sample concentrations were determined with $\leq 5 \%$ uncertainty $\left(2 \sigma_{\mathrm{m}}\right.$; external reproducibility of 8-16 measurements for $\mathrm{W}$, and 2-4 measurements for $\mathrm{Ba}$, Th and $\mathrm{U}$ ), though several analyses of phaneritic MORB glasses with low $\mathrm{W}$ concentrations resulted in uncertainties greater than $10 \%$. The accuracy of our laser ablation method is demonstrated in Fig. 1, where our Ba, Th, $\mathrm{U}$, and $\mathrm{W}$ measurements are compared with previously determined values from solution analyses, largely isotope dilution measurements.

\subsection{The best geochemical analogue to $W$}

Correlations between $\mathrm{Ba}$, Th, $\mathrm{U}$, and $\mathrm{W}$ concentrations in basaltic melts indicate that all four of these elements behave similarly during the evolution of basaltic magma. In order to establish which incompatible lithophile element most closely follows $\mathrm{W}$ in basaltic sources, and thus which $\mathrm{W} / \mathrm{X}$ ratio best represents a constant concentration ratio representative of the modern mantle, we can examine the constancy of each $\mathrm{W} / \mathrm{X}$ ratio across a wide range in $\mathrm{W}$ concentrations (Fig. 2). Sims and DePaolo (1997), however, noted that these types of diagrams have the distinct disadvantages that (a) the plotted variables are not statistically independent, and (b) variations in the $y$ variable of each curve (i.e., $\mathrm{W} / \mathrm{X}$, spanning $\sim 1$ order of magnitude) are disproportionately small in comparison to variations in the $x$-variable (i.e., W concentration, spanning $>2$ orders of magnitude). Additionally, the slope of each linear regression in Fig. 2 is sensitive to the relative value of the mean $\mathrm{W} / \mathrm{X}$ ratio, resulting in artificially high statistical deviations from a slope of 0 for $\mathrm{W} / \mathrm{Th}$ and $\mathrm{W} / \mathrm{U}$. Nevertheless, this plot serves the qualitative purpose of illustrating that $\mathrm{W} / \mathrm{Ba}, \mathrm{W} / \mathrm{Th}$ and $\mathrm{W} /$ $\mathrm{U}$ all remain relatively constant over a range of concentrations. In contrast, a ratio showing a systematic enrichment with increasing $\mathrm{W}$ concentration would indicate a lithophile element significantly less incompatible than $\mathrm{W}$ during mantle melting, and a systematic depletion would designate a more incompatible lithophile element.

A more quantitative way to evaluate which lithophile element behaves most analogously to $\mathrm{W}$ during silicate differentiation is through a log-log co-variation plot (Sims and DePaolo, 1997; Hofmann, 2003). Fig. 3 illustrates such a diagram, with the concentrations of $\mathrm{Ba}$, Th and $U$ plotted against the concentration of $\mathrm{W}$ in our basaltic samples. Using this diagnostic approach, a constant concentration ratio will yield a slope of unity $(m=1.00)$. According to our sample data, the linear regression that is statistically closest to unity is that between $\mathrm{W}$ and $\mathrm{U}$. Both Th and Ba yield slopes greater than unity when plotted against $\mathrm{W}$, implying a higher incompatibility of these elements in basaltic melts. Additionally, the $\mathrm{W} / \mathrm{U}$ ratio of the mantle does not appear to be affected by any differentiation processes, as the $\mathrm{W} / \mathrm{U}$ ratios of our samples are independent of $\mathrm{MgO}$ content as well as tectonic setting (Fig. 4). Accordingly, we conclude that $U$ is the incompatible lithophile element that behaves most similarly to $\mathrm{W}$ through silicate processing, despite the difference in mobility between these two elements in aqueous phases (Taylor and McLennan, 1985).

\section{Discussion}

\section{1. $W$ in the silicate Earth, modern mantle, and core}

Since $\mathrm{U}$ best mimics the geochemical behavior of $\mathrm{W}$ in our spectrum of basaltic samples, the average $\mathrm{W} / \mathrm{U}$ ratio of our dataset likely has not been affected by silicate differentiation events, and thus 
Table 2

Tungsten, $\mathrm{U}$, Th, and Ba sample concentration

\begin{tabular}{|c|c|c|c|c|c|c|c|c|c|c|c|c|c|c|c|c|}
\hline Sample/ID & Type $^{\mathrm{a}}$ & Location $^{\mathrm{b}}$ & W (ng/g) & $\pm 2 \sigma_{\mathrm{m}}{ }^{\mathrm{c}}$ & $\mathrm{U}(\mathrm{ng} / \mathrm{g})$ & $\pm 2 \sigma_{\mathrm{m}}^{\mathrm{c}}$ & Th $(\mathrm{ng} / \mathrm{g})$ & $\pm 2 \sigma_{\mathrm{m}}{ }^{\mathrm{c}}$ & $\mathrm{Ba}(\mu \mathrm{g} / \mathrm{g})$ & $\pm 2 \sigma_{\mathrm{m}}{ }^{\mathrm{c}}$ & $\mathrm{w} / \mathrm{U}$ & $2 \mathrm{RSD}^{\mathrm{d}}$ & $\mathrm{W} / \mathrm{Th}$ & $2 \mathrm{RSD}^{\mathrm{d}}$ & $\mathrm{W} / \mathrm{Ba}$ & $2 \mathrm{RSD}^{\mathrm{d}}$ \\
\hline OC180 D5-1 & N-MORB & NMAR & 13.84 & 0.51 & 19 & 5 & 51.2 & 6.7 & 2.18 & 0.02 & 0.732 & $29 \%$ & 0.270 & $14 \%$ & 0.00634 & $3.8 \%$ \\
\hline VG 6930 & N-MORB & NMAR & 23.45 & 1.36 & 17.2 & 0.2 & 53.3 & 0.9 & 3.26 & 0.12 & 1.365 & $5.9 \%$ & 0.440 & $6.0 \%$ & 0.00720 & $6.8 \%$ \\
\hline VG 6936 & N-MORB & NMAR & 20.99 & 0.69 & 16.9 & 0.2 & 58.9 & 0.5 & 3.36 & 0.08 & 1.246 & $3.5 \%$ & 0.357 & $3.4 \%$ & 0.00624 & $4.1 \%$ \\
\hline VG 6937 & N-MORB & NMAR & 27.48 & 1.15 & 20.2 & 0.5 & 77.3 & 2.9 & 5.17 & 0.21 & 1.362 & $4.9 \%$ & 0.356 & $5.6 \%$ & 0.00532 & $5.8 \%$ \\
\hline TK 5-1 & N-MORB & NMAR & 25.14 & 0.74 & 20.9 & 0.3 & 65.2 & 1.4 & 4.04 & 0.03 & 1.205 & $3.3 \%$ & 0.386 & $3.7 \%$ & 0.00622 & $3.0 \%$ \\
\hline OCD180 D26-3 & N-MORB & NMAR & 21.1 & 0.2 & 53.9 & 1.0 & 190 & 8 & 10.4 & 0.0 & 0.390 & $2.0 \%$ & 0.111 & $4.3 \%$ & 0.00202 & $0.9 \%$ \\
\hline TK $1-2$ & N-MORB & NMAR & 34.21 & 0.69 & 36.8 & 0.9 & 130 & 7 & 5.57 & 0.03 & 0.930 & $3.2 \%$ & 0.262 & $5.6 \%$ & 0.00614 & $2.1 \%$ \\
\hline TK 2-1 & N-MORB & NMAR & 12.7 & 0.3 & 41.8 & 0.6 & 151 & 9 & 6.82 & 0.03 & 0.304 & $2.4 \%$ & 0.084 & $6.1 \%$ & 0.00186 & $2.0 \%$ \\
\hline OC180 D13-2 & N-MORB & NMAR & 28.9 & 0.4 & 53.9 & 1.9 & 200 & 3 & 12.6 & 0.13 & 0.537 & $3.8 \%$ & 0.145 & $2.0 \%$ & 0.00230 & $1.7 \%$ \\
\hline VG 0738 & N-MORB & NMAR & 13.8 & 0.3 & 45.8 & 1.5 & 181 & 9 & 8.02 & 0.30 & 0.301 & $4.2 \%$ & 0.076 & $5.7 \%$ & 0.00172 & $4.5 \%$ \\
\hline VG 0739 & N-MORB & NMAR & 13.2 & 0.7 & 50.6 & 0.7 & 164 & 2 & 7.94 & 0.40 & 0.262 & $5.2 \%$ & 0.080 & $5.1 \%$ & 0.00167 & $7 \%$ \\
\hline TK 4-6 & N-MORB & NMAR & 15.7 & 0.2 & 48.2 & 0.9 & 137 & 5 & 8.43 & 0.03 & 0.326 & $2.3 \%$ & 0.114 & $3.7 \%$ & 0.00186 & $1.3 \%$ \\
\hline VG 0741 & N-MORB & NMAR & 16.2 & 0.4 & 55.9 & 1.6 & 179 & 7 & 8.75 & 0.15 & 0.290 & $3.9 \%$ & 0.091 & $4.9 \%$ & 0.00185 & $3.2 \%$ \\
\hline VG 0735 & N-MORB & NMAR & 22.2 & 0.4 & 65.1 & 0.7 & 234 & 7 & 11.6 & 0.38 & 0.342 & $1.9 \%$ & 0.095 & $3.4 \%$ & 0.00192 & $3.7 \%$ \\
\hline Atlantica & E-MORB & MAR & 109.7 & 1.6 & 160 & 4 & 713 & 11 & 64.3 & 1.6 & 0.685 & $3.0 \%$ & 0.154 & $2.2 \%$ & 0.00171 & $3.0 \%$ \\
\hline MAR & N-MORB & MAR & 39.74 & 0.61 & 31.0 & 0.6 & 109 & 2 & 5.93 & 0.05 & 1.283 & $2.6 \%$ & 0.364 & $2.4 \%$ & 0.00670 & $1.7 \%$ \\
\hline VG 9055 & N-MORB & SMAR & 16.06 & 0.37 & 35.2 & 0.6 & 105 & 3 & 2.89 & 0.07 & 0.456 & $2.8 \%$ & 0.152 & $3.3 \%$ & 0.00555 & $3.4 \%$ \\
\hline VG 9056 & N-MORB & SMAR & 16.08 & 0.83 & 35.7 & 2.5 & 114 & 2 & 2.94 & 0.13 & 0.451 & $8.6 \%$ & 0.142 & $5.5 \%$ & 0.00547 & $7 \%$ \\
\hline VG 9052 & N-MORB & SMAR & 15.39 & 0.68 & 32.7 & 0.1 & 101 & 2 & 2.99 & 0.09 & 0.470 & $4.5 \%$ & 0.152 & $4.7 \%$ & 0.00514 & $5.3 \%$ \\
\hline VG 3630 & E-MORB & SMAR & 47.3 & 0.6 & 165 & 2 & 508 & 31 & 29.9 & 1.6 & 0.287 & $1.9 \%$ & 0.093 & $6.3 \%$ & 0.00158 & $5 \%$ \\
\hline R74-6 & N-MORB & EPR & 10.19 & 0.67 & 10.30 & 0.39 & 23.4 & 0.6 & 0.88 & 0.06 & 0.989 & $7.6 \%$ & 0.435 & $7.1 \%$ & 0.01157 & $9.6 \%$ \\
\hline $2384-9$ & N-MORB & EPR & 8.7 & 0.3 & 15.2 & 0.5 & 33 & 1 & 1.42 & 0.09 & 0.571 & $5.0 \%$ & 0.264 & $3.9 \%$ & 0.00611 & $7 \%$ \\
\hline $2384-3$ & N-MORB & EPR & 20.31 & 1.05 & 15.2 & 0.7 & 30.0 & 0.8 & 1.38 & 0.12 & 1.339 & $6.7 \%$ & 0.676 & $5.8 \%$ & 0.01467 & $10 \%$ \\
\hline R30-30 & N-MORB & EPR & 29.7 & 4.6 & 32.5 & 1.6 & 80.9 & 3.6 & 1.80 & 0.15 & 0.914 & $16 \%$ & 0.368 & $16 \%$ & 0.01656 & $18 \%$ \\
\hline R1-14 & N-MORB & EPR & 14.4 & 1.66 & 17.4 & 1.6 & 42.2 & 1.6 & 1.72 & 0.13 & 0.827 & $15 \%$ & 0.340 & $12 \%$ & 0.00834 & $14 \%$ \\
\hline $2387-2$ & N-MORB & EPR & 13.2 & 0.2 & 35.2 & 0.6 & 105 & 2 & 7.34 & 0.21 & 0.376 & $2.2 \%$ & 0.126 & $2.8 \%$ & 0.00180 & $3.2 \%$ \\
\hline R28-7 & N-MORB & EPR & 15.8 & 2.19 & 25.5 & 0.5 & 60.3 & 2.3 & 1.86 & 0.09 & 0.619 & $14 \%$ & 0.261 & $14 \%$ & 0.00848 & $15 \%$ \\
\hline $2706-7$ & N-MORB & EPR & 34.00 & 0.98 & 37.2 & 0.4 & 84.4 & 1.0 & 4.91 & 0.12 & 0.913 & $3.1 \%$ & 0.403 & $3.1 \%$ & 0.00692 & $3.7 \%$ \\
\hline R501-7 & E-MORB & EPR & 37.4 & 0.5 & 92.5 & 0.6 & 311 & 9 & 25.2 & 0.2 & 0.405 & $1.5 \%$ & 0.120 & $3.3 \%$ & 0.00148 & $1.6 \%$ \\
\hline R494-5 & E-MORB & EPR & 35.4 & 0.4 & 87.1 & 3.0 & 269 & 5 & 24.7 & 0.7 & 0.407 & $3.6 \%$ & 0.132 & $2.4 \%$ & 0.00143 & $3.1 \%$ \\
\hline R75-2 & E-MORB & EPR & 138 & 5 & 424 & 17 & $1.15 E+03$ & $3.7 E+01$ & 53.6 & 1.3 & 0.325 & $5.2 \%$ & 0.120 & $4.7 \%$ & 0.00257 & $4.2 \%$ \\
\hline R501-2 & E-MORB & EPR & 43.5 & 0.5 & 103.7 & 2.1 & 336 & 6 & 27.1 & 0.4 & 0.420 & $2.3 \%$ & 0.129 & $2.0 \%$ & 0.00160 & $1.9 \%$ \\
\hline R93-7 & E-MORB & EPR & 51.0 & 0.9 & 137 & 0 & 476 & 13 & 22.3 & 0.4 & 0.374 & $1.8 \%$ & 0.107 & $3.2 \%$ & 0.00228 & $2.5 \%$ \\
\hline R15-2 & E-MORB & EPR & 555 & 8 & 990 & 41 & $3.58 \mathrm{E}+03$ & $1.4 \mathrm{E}+02$ & 306 & 7 & 0.560 & $4.4 \%$ & 0.155 & $4.1 \%$ & 0.00181 & $2.8 \%$ \\
\hline R78-6 & E-MORB & EPR & 473 & 14 & 716 & 9 & $2.87 \mathrm{E}+03$ & $1.1 \mathrm{E}+02$ & 207 & 5 & 0.660 & $3.3 \%$ & 0.165 & $5.0 \%$ & 0.00229 & $3.8 \%$ \\
\hline VE-32 & E-MORB & EPR & 56.8 & 0.6 & 122 & 3 & 353 & 12 & 51.5 & 0.4 & 0.465 & $2.7 \%$ & 0.161 & $3.6 \%$ & 0.00110 & $1.4 \%$ \\
\hline 2079-2 & E-MORB & JdF Ridge & 23.7 & 0.3 & 78.7 & 0.8 & 244 & 13 & 14.0 & 0.1 & 0.302 & $1.6 \%$ & 0.097 & $5.3 \%$ & 0.00169 & $1.5 \%$ \\
\hline $2082-6 \mathrm{~A}$ & E-MORB & JdF Ridge & 21.7 & 0.3 & 69.4 & 1.4 & 166 & 9 & 12.4 & 0.2 & 0.313 & $2.5 \%$ & 0.131 & $5.6 \%$ & 0.00175 & $2.1 \%$ \\
\hline JdF MORB & E-MORB & JdF Ridge & 31.2 & 0.3 & 104 & 1 & 249 & 5 & 15.7 & 0.9 & 0.300 & $1.6 \%$ & 0.125 & $2.0 \%$ & 0.00198 & $6 \%$ \\
\hline VG-2 & E-MORB & JdF Ridge & 31.2 & 0.6 & 110.9 & 2.1 & 348 & 9 & 17.5 & 0.4 & 0.282 & $2.8 \%$ & 0.090 & $3.4 \%$ & 0.00178 & $2.9 \%$ \\
\hline VG 5256 & N-MORB & N Indian Ridge & 9.59 & 0.39 & 10.37 & 0.49 & 42.5 & 3.6 & 2.02 & 0.04 & 0.925 & $6.2 \%$ & 0.225 & $9.3 \%$ & 0.00475 & $4.4 \%$ \\
\hline VG 5253 & N-MORB & N Indian Ridge & 11.50 & 1.09 & 10.92 & 0.40 & 36.0 & 2.5 & 1.94 & 0.02 & 1.053 & $10 \%$ & 0.319 & $12 \%$ & 0.00593 & $9.5 \%$ \\
\hline VG 5252 & N-MORB & N Indian Ridge & 11.81 & 0.92 & 11.3 & 0.5 & 35.6 & 1.0 & 2.06 & 0.10 & 1.049 & $8.8 \%$ & 0.332 & $8.3 \%$ & 0.00573 & $9 \%$ \\
\hline VG 5259 & N-MORB & N Indian Ridge & 9.73 & 0.75 & 10.36 & 0.56 & 35.7 & 1.6 & 2.01 & 0.08 & 0.939 & $9.4 \%$ & 0.272 & $9.0 \%$ & 0.00485 & $9 \%$ \\
\hline MD37-18 D2 & E-MORB & SE Indian Ridge & 149 & 5 & 185 & 11 & 858 & 9 & 55.0 & 1.5 & 0.805 & $6.9 \%$ & 0.174 & $3.2 \%$ & 0.00271 & $4.2 \%$ \\
\hline
\end{tabular}


Table 2 (continued)

\begin{tabular}{|c|c|c|c|c|c|c|c|c|c|c|c|c|c|c|c|c|}
\hline Sample/ID & Type $^{a}$ & Location $^{\mathrm{b}}$ & W (ng/g) & $\pm 2 \sigma_{\mathrm{m}}{ }^{\mathrm{c}}$ & $\mathrm{U}(\mathrm{ng} / \mathrm{g})$ & $\pm 2 \sigma_{\mathrm{m}}{ }^{\mathrm{c}}$ & Th $(\mathrm{ng} / \mathrm{g})$ & $\pm 2 \sigma_{\mathrm{m}}{ }^{\mathrm{c}}$ & $\mathrm{Ba}(\mu \mathrm{g} / \mathrm{g})$ & $\pm 2 \sigma_{\mathrm{m}}{ }^{\mathrm{c}}$ & $\mathrm{W} / \mathrm{U}$ & $2 \mathrm{RSD}^{\mathrm{d}}$ & $\mathrm{W} / \mathrm{Th}$ & $2 \mathrm{RSD}^{\mathrm{d}}$ & W/Ba & $2 \mathrm{RSD}^{\mathrm{d}}$ \\
\hline AAD $10-10$ & E-MORB & SE Indian Ridge & 25.2 & 0.4 & 55.5 & 0.9 & 241 & 5 & 14.8 & 0.3 & 0.454 & $2.3 \%$ & 0.105 & $2.7 \%$ & 0.00170 & $2.6 \%$ \\
\hline AAD 7-3 & E-MORB & SE Indian Ridge & 64.4 & 0.4 & 133 & 1 & 557 & 9 & 33.5 & 0.7 & 0.485 & $1.0 \%$ & 0.115 & $1.7 \%$ & 0.00192 & $2.1 \%$ \\
\hline AAD 8-8 & E-MORB & SE Indian Ridge & 130 & 2 & 191 & 5 & 592 & 20 & 68.2 & 1.4 & 0.678 & $3.0 \%$ & 0.219 & $3.6 \%$ & 0.00190 & $2.4 \%$ \\
\hline MD 23-18 S3 & N-MORB & C Indian Ridge & 6.27 & 0.34 & 5.24 & 0.14 & 24.3 & 2.1 & 1.18 & 0.07 & 1.196 & $6.0 \%$ & 0.258 & $10 \%$ & 0.00534 & $8 \%$ \\
\hline JC 2-17 D1(3) & E-MORB & C Indian Ridge & 22.8 & 0.7 & 52.2 & 2.2 & 211 & 4 & 15.6 & 0.1 & 0.437 & $5.3 \%$ & 0.108 & $3.8 \%$ & 0.00146 & $3.3 \%$ \\
\hline JC 3-02 D1 & E-MORB & SW Indian Ridge & 34.4 & 0.7 & 94.4 & 2.5 & 356 & 6 & 23.1 & 0.9 & 0.365 & $3.3 \%$ & 0.097 & $2.6 \%$ & 0.00149 & $4.3 \%$ \\
\hline MD 34 D5 & E-MORB & SW Indian Ridge & 26.0 & 0.4 & 39.5 & 1.0 & 204 & 8 & 63.0 & 0.4 & 0.657 & $2.9 \%$ & 0.127 & $4.2 \%$ & 0.00041 & $1.6 \%$ \\
\hline Pu'u O'o 90 & OIB & Kilauea, $\mathrm{HI}$ & 161 & 7 & 278 & 9 & $1.13 E+03$ & $4.5 \mathrm{E}+01$ & 108 & 8 & 0.579 & $5.5 \%$ & 0.143 & $6.0 \%$ & 0.00149 & $9 \%$ \\
\hline Pu'u O'o 93 & OIB & Kilauea, HI & 177 & 4 & 310 & 0 & $8.93 \mathrm{E}+02$ & $2.1 \mathrm{E}-01$ & 110 & 2 & 0.572 & $2.0 \%$ & 0.199 & $2.0 \%$ & 0.00161 & $2.7 \%$ \\
\hline VG A-99 & OIB & Kilauea, HI & 248 & 4 & 500 & 13 & $1.97 \mathrm{E}+03$ & $5.5 \mathrm{E}+01$ & 184 & 6 & 0.495 & $3.1 \%$ & 0.126 & $3.3 \%$ & 0.00134 & $3.7 \%$ \\
\hline NMNH 116111-22 & OIB & Kilauea, HI & 228 & 8 & 351 & 5 & $1.35 \mathrm{E}+03$ & $1.5 \mathrm{E}+01$ & 123 & 2 & 0.651 & $3.9 \%$ & 0.170 & $3.8 \%$ & 0.00186 & $4.1 \%$ \\
\hline NMNH 113154-555 & OIB & Galapagos & 19.4 & 0.5 & 23.3 & 0.3 & 104 & 3 & 6.82 & 0.14 & 0.832 & $2.9 \%$ & 0.186 & $4.1 \%$ & 0.00284 & $3.3 \%$ \\
\hline NMNH 113154-557 & OIB & Galapagos & 27.5 & 0.5 & 48.1 & 0.8 & 213 & 7 & 12.5 & 0.4 & 0.573 & $2.3 \%$ & 0.129 & $3.7 \%$ & 0.00221 & $3.5 \%$ \\
\hline NMNH 113710-F709 & OIB & Galapagos & 218 & 4 & 400 & 11 & $1.64 \mathrm{E}+03$ & $4.9 \mathrm{E}+01$ & 114 & 13 & 0.545 & $3.3 \%$ & 0.133 & $3.6 \%$ & 0.00191 & $12 \%$ \\
\hline NMNH 112048-2 & OIB & Hekla, Iceland & 766 & 31 & 1084 & 12 & $4.96 \mathrm{E}+03$ & $4.8 \mathrm{E}+01$ & 325 & 1 & 0.707 & $4.3 \%$ & 0.154 & $4.2 \%$ & 0.00236 & $4.1 \%$ \\
\hline NMNH 112055 & OIB & Hekla, Iceland & 828 & 24 & $1.22 \mathrm{E}+03$ & $2.1 \mathrm{E}+01$ & $5.45 \mathrm{E}+03$ & $1.5 \mathrm{E}+02$ & 335 & 12 & 0.679 & $3.4 \%$ & 0.152 & $3.9 \%$ & 0.00247 & $4.5 \%$ \\
\hline NMNH 112207-75 & OIB & Heard Is., Australia & 675 & 6 & 1033 & 14 & $4.80 \mathrm{E}+03$ & $4.6 \mathrm{E}+02$ & 545 & 3 & 0.653 & $1.6 \%$ & 0.141 & $9.5 \%$ & 0.00124 & $1.0 \%$ \\
\hline NMNH 113057-3 & ICV & Nyiragongo, Zaire & 2144 & 30 & 7900 & 51 & $2.35 \mathrm{E}+04$ & $2.0 \mathrm{E}+02$ & 2259 & 22 & 0.271 & $1.5 \%$ & 0.091 & $1.6 \%$ & 0.00095 & $1.7 \%$ \\
\hline NMNH 115693 & ICV & Nyiragongo, Zaire & 2408 & 105 & 7510 & 126 & $2.18 \mathrm{E}+04$ & $1.2 \mathrm{E}+03$ & 2157 & 152 & 0.321 & $4.7 \%$ & 0.111 & $7.0 \%$ & 0.00112 & $8.3 \%$ \\
\hline NMNH 12514 & ICV & Nyamuragira, Zaire & 1457 & 30 & 2003 & 30 & $1.43 \mathrm{E}+04$ & $3.4 \mathrm{E}+02$ & 1021 & 54 & 0.727 & $2.5 \%$ & 0.102 & $3.1 \%$ & 0.00143 & $5.7 \%$ \\
\hline NMNH 068376 & ICV & Sunset Crater, AZ & 316 & 13 & 927 & 53 & $3.72 E+03$ & $1.8 \mathrm{E}+02$ & 453 & 25 & 0.341 & $7.0 \%$ & 0.085 & $6.2 \%$ & 0.00070 & $6.8 \%$ \\
\hline $42995-3 C$ & ICV & Yucca Mtn., NV & 487 & 19 & 2103 & 71 & $1.06 E+04$ & $7.2 \mathrm{E}+02$ & 1511 & 90 & 0.232 & $5.1 \%$ & 0.046 & $7.8 \%$ & 0.00032 & $7.1 \%$ \\
\hline NMNH 116605.0013 & ICV & Tower Hill, Australia & 943 & 40 & 1174 & 32 & $6.61 \mathrm{E}+03$ & $1.5 \mathrm{E}+02$ & 503 & 11 & 0.803 & $5.1 \%$ & 0.143 & $4.9 \%$ & 0.00187 & $4.8 \%$ \\
\hline NMNH 108800 & $\mathrm{IAB}$ & Oshima Is., Japan & 258 & 4 & 141 & 2 & $3.03 \mathrm{E}+02$ & $8.6 \mathrm{E}+00$ & 206 & 8 & 1.828 & $2.1 \%$ & 0.853 & $3.2 \%$ & 0.00125 & $4.3 \%$ \\
\hline NMNH 116701-2 & $\mathrm{IAB}$ & Galunggung, Indonesia & 397 & 31 & 246 & 6 & $1.50 \mathrm{E}+03$ & $2.2 \mathrm{E}+02$ & 115 & 21 & 1.613 & $8.1 \%$ & 0.265 & $16.5 \%$ & 0.00345 & $19.8 \%$ \\
\hline NMNH 116141 & $\mathrm{CAB}$ & Pacaya, Guatemala & 329 & 8 & 930 & 45 & $3.17 \mathrm{E}+03$ & $3.8 \mathrm{E}+02$ & 684 & 18 & 0.354 & $5.4 \%$ & 0.104 & $12.3 \%$ & 0.00048 & $3.5 \%$ \\
\hline NMNH 108135 & $\mathrm{CAB}$ & Mexico & 201 & 5 & 295 & 13 & $1.06 \mathrm{E}+03$ & $1.1 \mathrm{E}+02$ & 285 & 26 & 0.681 & $5.2 \%$ & 0.190 & $10.2 \%$ & 0.00070 & $9.6 \%$ \\
\hline NMNH 116601-12 & $\mathrm{CAB}$ & Durango, Mexico & 1101 & 16 & 1352 & 39 & $6.54 \mathrm{E}+03$ & $1.7 \mathrm{E}+02$ & 516 & 12 & 0.814 & $3.2 \%$ & 0.168 & $3.0 \%$ & 0.00213 & $2.7 \%$ \\
\hline NMNH 113559-1 & $\mathrm{CAB}$ & Vesuvius, Italy & 926 & 23 & 2893 & 223 & $1.12 \mathrm{E}+04$ & $7.5 \mathrm{E}+02$ & 739 & 61 & 0.320 & $8.1 \%$ & 0.082 & $7.2 \%$ & 0.00125 & $8.6 \%$ \\
\hline VG 3636 & BABB & Scotia Back-Arc & 45 & 1 & 109 & 2 & $3.76 \mathrm{E}+02$ & $2.9 \mathrm{E}+00$ & 41 & 1 & 0.411 & $2.7 \%$ & 0.119 & $1.6 \%$ & 0.00109 & $1.9 \%$ \\
\hline VG 3637 & BABB & Scotia Back-Arc & 49 & 1 & 112 & 1 & $4.04 \mathrm{E}+02$ & $4.9 \mathrm{E}+00$ & 43 & 0 & 0.434 & $1.7 \%$ & 0.120 & $1.6 \%$ & 0.00113 & $1.6 \%$ \\
\hline VG 3635 & BABB & Scotia Back-Arc & 46 & 0 & 106 & 1 & 431 & 6 & 40 & 1 & 0.433 & $1.4 \%$ & 0.107 & $1.7 \%$ & 0.00116 & $1.9 \%$ \\
\hline VG 3638 & BABB & Scotia Back-Arc & 53 & 1 & 107 & 2 & $3.71 E+02$ & $3.9 \mathrm{E}+00$ & 54 & 2 & 0.491 & $1.9 \%$ & 0.142 & $1 \%$ & 0.00098 & $4 \%$ \\
\hline VG 3634 & BABB & Scotia Back-Arc & 43.5 & 0.6 & 102.5 & 3.3 & 398 & 6 & 38.4 & 0.9 & 0.424 & $3.5 \%$ & 0.109 & $2.0 \%$ & 0.00113 & $2.6 \%$ \\
\hline SLB 133 GA & BABB & Southern Lau Basin & 68.9 & 0.5 & 198.5 & 1.7 & 463 & 3 & 110.0 & 3.1 & 0.347 & $1.2 \%$ & 0.149 & $1.0 \%$ & 0.00063 & $2.9 \%$ \\
\hline SLB 58 GA-1 & BABB & Southern Lau Basin & 71.9 & 0.9 & 200.4 & 2.4 & 484 & 3 & 94.5 & 3.0 & 0.359 & $1.7 \%$ & 0.148 & $1.4 \%$ & 0.00076 & $3.4 \%$ \\
\hline SLB 53 GC-1 & BABB & Southern Lau Basin & 53.3 & 0.6 & 151.8 & 1.0 & 347 & 11 & 79.7 & 3.7 & 0.351 & $1.3 \%$ & 0.154 & $3.3 \%$ & 0.00067 & $4.8 \%$ \\
\hline SLB 127 KD & $\mathrm{BABB}$ & Southern Lau Basin & 71.5 & 1.5 & 185.4 & 2.4 & 451 & 13 & 95.0 & 2.7 & 0.386 & $2.5 \%$ & 0.159 & $3.7 \%$ & 0.00075 & $3.5 \%$ \\
\hline CLB 7GC & BABB & Central Lau Basin & 38.1 & 1.7 & 22 & 0 & 79 & 4 & 6 & 0 & 1.762 & $4.6 \%$ & 0.485 & $6.9 \%$ & 0.00613 & $4.9 \%$ \\
\hline CLB 42GC & BABB & Central Lau Basin & 32.6 & 0.6 & 21 & 0 & 69 & 1 & 6 & 0 & 1.588 & $2.1 \%$ & 0.471 & $2.0 \%$ & 0.00506 & $3.0 \%$ \\
\hline CLB 46GC & BABB & Central Lau Basin & 14.0 & 0.1 & 31 & 1 & 105 & 1 & 8.0 & 0.2 & 0.449 & $1.9 \%$ & 0.133 & $1.7 \%$ & 0.00175 & $3.1 \%$ \\
\hline \multicolumn{17}{|l|}{ Hawaiian Picrites } \\
\hline Lo-02-02 & & & 265 & 4 & 337 & 11 & $1.14 E+03$ & $3.6 \mathrm{E}+01$ & 147 & 4 & 0.786 & $3.5 \%$ & 0.232 & $3.6 \%$ & 0.00180 & $3.4 \%$ \\
\hline Lo-02-04 & & & 313 & 6 & 365 & 7 & $1.27 \mathrm{E}+03$ & $1.8 \mathrm{E}+01$ & 156 & 5 & 0.857 & $2.7 \%$ & 0.246 & $2.3 \%$ & 0.00201 & $3.5 \%$ \\
\hline $\mathrm{H}-11$ & & & 113 & 2 & 159 & 4 & 533 & 13 & 56.0 & 1.7 & 0.710 & $2.9 \%$ & 0.211 & $3.1 \%$ & 0.00201 & $3.6 \%$ \\
\hline
\end{tabular}

a $\mathrm{N}$-MORB, E-MORB, OIB, and BABB defined in text; ICV = intracontinental basalt, IAB = island arc basalt, $C A B=$ continental arc basalt.

b NMAR = North Mid-Atlantic Ridge, SMAR $=$ South Mid-Atlantic Ridge, EPR $=$ East Pacific Rise, JdF Ridge $=$ Juan de Fuca Ridge.

d Relative uncertainties $(2 \sigma)$ in W/X ratios were determined through error propagation according to Eq. 3.26 from Bevington and Robinson (2003). 


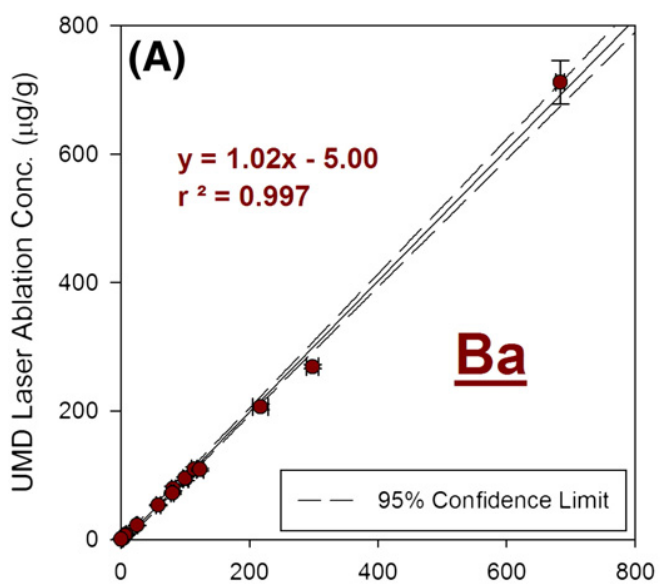

Previously Published Conc. $(\mu \mathrm{g} / \mathrm{g})$

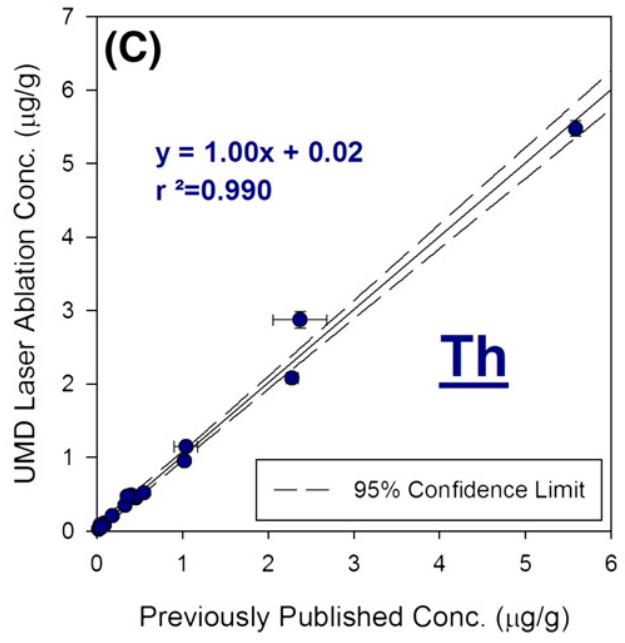

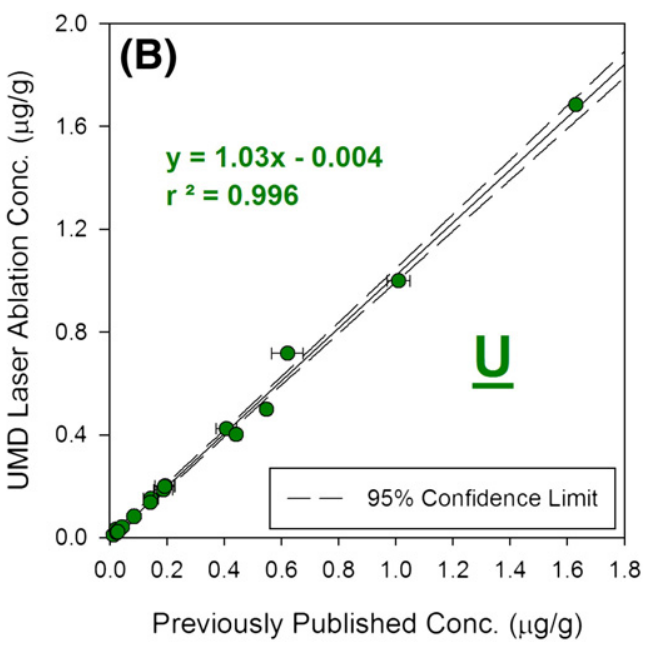

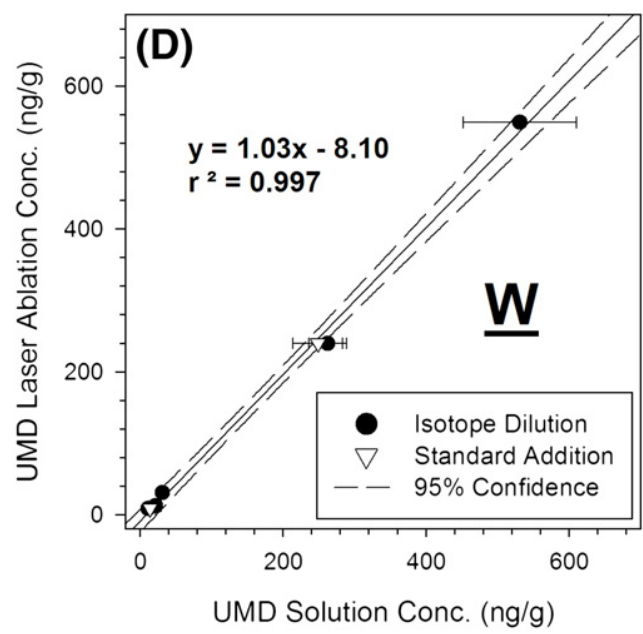

Fig. 1. Correlations between our LA-ICP-MS concentration data and those of previously published and/or recently acquired solution analyses of the same samples. Previously published values of Ba, Th and U include isotope dilution measurements from Loock et al. (1990), solution ICP-MS measurements from Niu and Batiza (1997), and isotope dilution analyses of the homogeneous MPI-DING and USGS reference glasses by Jochum et al. (2005, 2006). In regards to W, both natural samples (2384-9 and JdF MORB) and geologic reference glasses (BIR-1g, BHVO-2g, and BCR-2g) were analyzed by laser ablation and solution ICP-MS at the University of Maryland, including via both isotope dilution and highprecision standard addition methods. Details of these measurements are provided in the Supplementary materials. In all cases, our laser measurements agree well with solution measurements (within $\pm 3 \%$ ) over several orders of magnitude for each element. Deviations from a perfect correlation with slope $m=1.00$ can be accounted for by the uncertainty in each of our measurements, which typically range from $2-5 \%\left(2 \sigma_{\mathrm{m}}\right)$.

represents the $\mathrm{W} / \mathrm{U}$ ratio of the modern mantle. The average $\mathrm{W} / \mathrm{U}$ ratio of our comprehensive sample suite, and thus of the mantle, is $0.65 \pm 0.45(2 \sigma ; n=86)$, which is statistically indistinguishable from the value obtained considering only $\mathrm{MORB}\left(\mathrm{W} / \mathrm{U}_{\mathrm{MORB}}=0.65 \pm 0.41\right.$, $n=52)$, OIB $\left(\mathrm{W} / \mathrm{U}_{\mathrm{OIB}}=0.63 \pm 0.07, n=10\right)$, or $\mathrm{BABB}\left(\mathrm{W} / \mathrm{U}_{\mathrm{BABB}}=0.62 \pm 0.09\right.$, $n=12)$.

The $\mathrm{W} / \mathrm{U}$ ratio of the modern mantle is also coincident with that of the continental crust $(\mathrm{W} / \mathrm{U}=0.77 \pm 0.33,2 \sigma$; Rudnick and Gao, 2003), which is largely based on upper-crustal sediments and loess deposits (median $\mathrm{W} / \mathrm{U} \approx 0.8$ ) and high-grade metamorphic terrains representative of the lower crust (median $\mathrm{W} / \mathrm{U} \approx 0.8$; data from Newsom et al., 1996 and references cited therein). Therefore, the $\mathrm{W} / \mathrm{U}$ ratio of the mantle may also be inferred to be representative of the entire silicate portion of the Earth, assuming the absence of a "hidden" repository with a $\mathrm{W} / \mathrm{U}$ ratio outside the range displayed by sampled reservoirs (McDonough, 1991; Lassiter, 2004; Boyet and Carlson, 2005; Tolstikhin and Hofmann, 2005; Boyet and Carlson, 2006; Tolstikhin et al., 2006). Assuming a U concentration of $20 \pm 8(2 \sigma) \mathrm{ng} / \mathrm{g}$ in the bulk silicate Earth (McDonough and Sun, 1995; Palme and O'Neill, 2003; Lyubetskaya and Korenaga, 2007) and a W/U ratio of $0.65 \pm 0.45(2 \sigma)$, the concentration of $\mathrm{W}$ in the silicate portion of the Earth is $13 \pm 10 \mathrm{ng} / \mathrm{g}$.

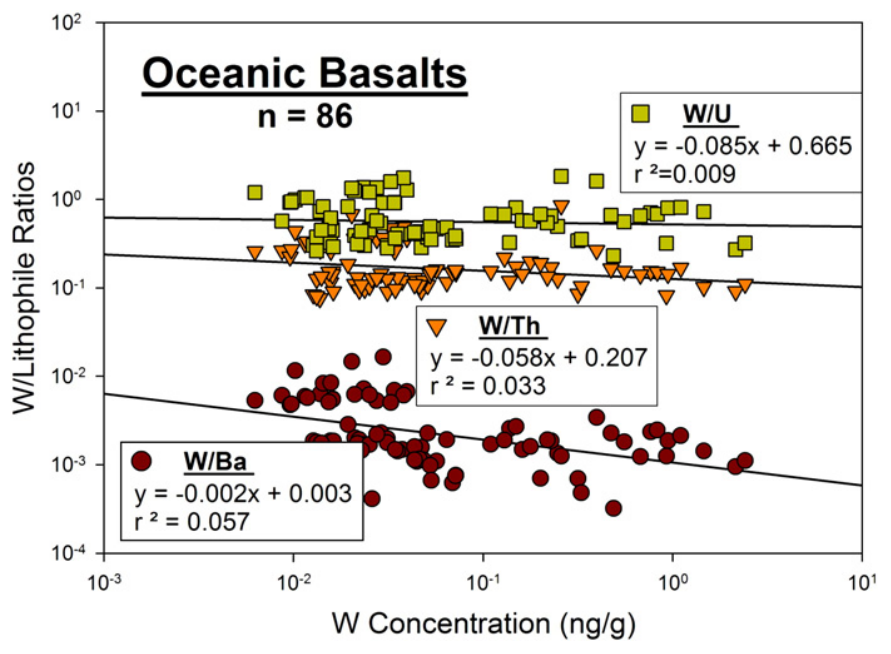

Fig. 2. $\mathrm{W} / \mathrm{Ba}, \mathrm{W} / \mathrm{Th}$ and $\mathrm{W} / \mathrm{U}$ ratios plotted against the $\mathrm{W}$ concentration $(\mathrm{ng} / \mathrm{g})$ of our samples. A perfectly constant element ratio would yield a slope of zero. The accompanying statistics suggest a systematic decrease in both $W / T h$ and $W / U$ as a function of increasing $\mathrm{W}$ concentration, but this represents an artificial bias due to the higher absolute value of the $y$-variables of these curves compared to $\mathrm{W} / \mathrm{Ba}$. 


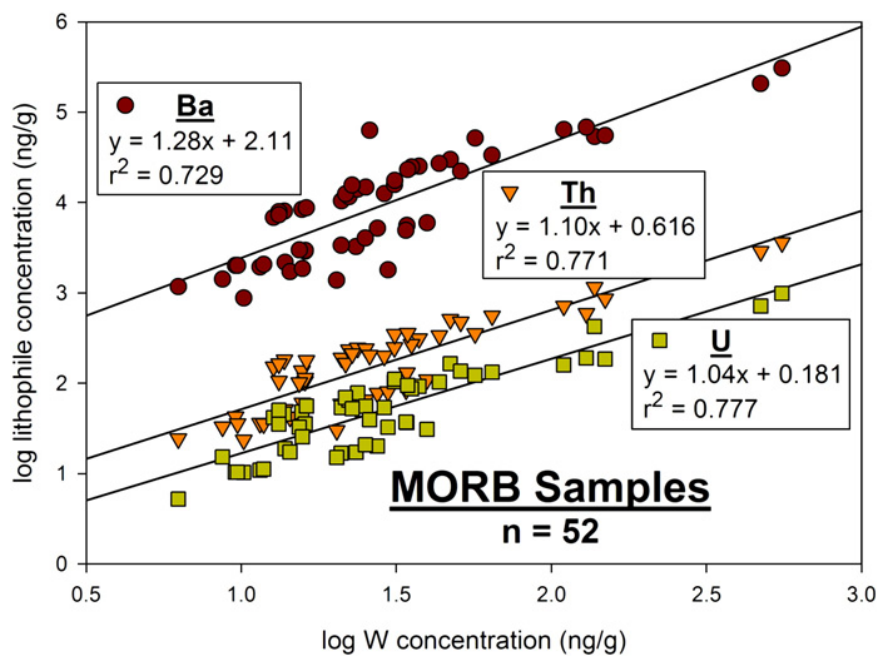

Fig. 3. Log-log co-variation diagram plotting the concentrations (ng/g) of Ba, Th and versus the concentration of $\mathrm{W}$ in our MORB samples. On this type of plot, because the plotted samples share the same mantle source region (the depleted MORB mantle, or DMM), a regression line of slope 1.00 represents a perfectly constant element ratio, whereas a slope of $>1.00$ indicates a more incompatible lithophile element (e.g., Ba, Th and $\mathrm{U}$ ) during mantle melting. It can be seen that the linear regression between $\mathrm{W}$ and $\mathrm{U}$ yields the slope nearest unity ( $m=1.04 \pm 0.18,95 \%$ confidence) and with the least statistical scatter $\left(r^{2}=0.777\right)$. Although both $\mathrm{Ba}$ and Th yield slopes greater than unity, suggesting that they are more incompatible than $\mathrm{W}$ during mantle melting, the slope of $\log$ Th versus $\log \mathrm{W}$ is within uncertainty of unity ( $m=1.10 \pm 0.20,95 \%$ confidence).

Newsom et al. (1996) and Rudnick and Gao (2003) reported that the bulk continental crust ( $\sim 0.5 \%$ of the bulk silicate Earth, by mass) contains approximately $1000 \pm 300(2 \sigma) \mathrm{ng} / \mathrm{g} \mathrm{W}$. Based on mass balance contributions and our preferred estimate of $\mathrm{W}$ in the silicate Earth, the mean concentration of $\mathrm{W}$ in the Earth's modern mantle reservoir is calculated to be $8.3 \pm 7.1 \mathrm{ng} / \mathrm{g}$ (with MORB and OIB representing depleted and enriched source regions, respectively). Based on the unweighted mean $\mathrm{W} / \mathrm{Hf}$ ratio of carbonaceous chondrites (W/ $\mathrm{Hf}=0.91 \pm 0.06,2 \sigma_{\mathrm{m}}$; Kleine et al., 2004b), a silicate Earth composition with $280 \pm 60(2 \sigma) \mathrm{ng} / \mathrm{g} \mathrm{Hf}$, and negligible Hf in the core, the bulk Earth contains $172 \pm 36 \mathrm{ng} / \mathrm{g} \mathrm{W}$. Accordingly, simple core-modeling and mass balance considerations predict that the core has $500 \pm 120 \mathrm{ng} / \mathrm{g} \mathrm{W}$; this indicates a W metal/silicate partition coefficient $\left(D^{\text {metal/silicate }}\right)$ of $\sim 40$

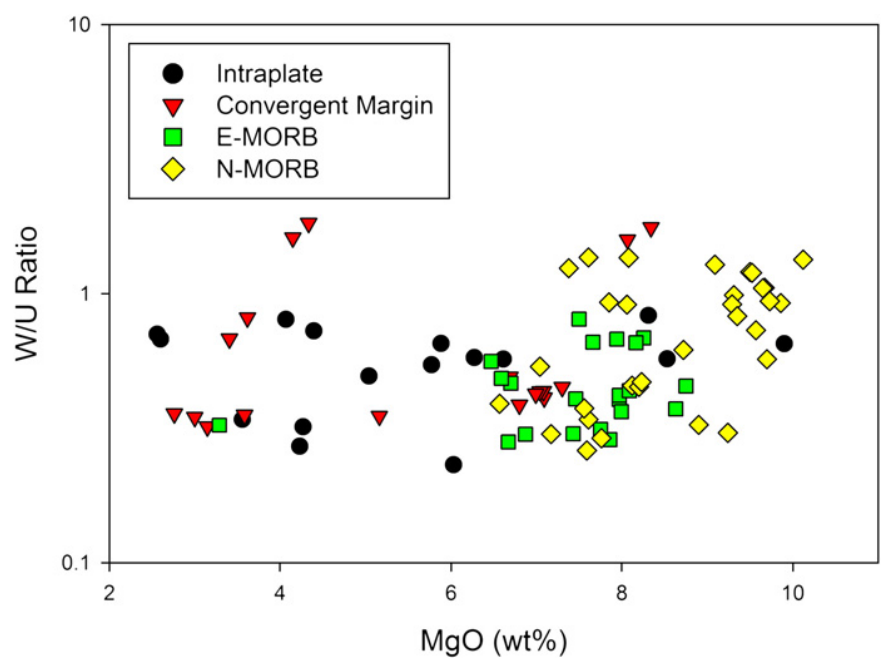

Fig. 4. The $\mathrm{W} / \mathrm{U}$ ratio of our samples plotted against $\mathrm{MgO}$ (wt.\%) content. The $\mathrm{W} / \mathrm{U}$ ratios of our modern basalts appear to be independent of the degree of silicate processing, and no systematic differences among the different sample populations (including N-MORB E-MORB, convergent margin volcanics, and intraplate basalts) can be seen with regards to $\mathrm{W} / \mathrm{U}$.

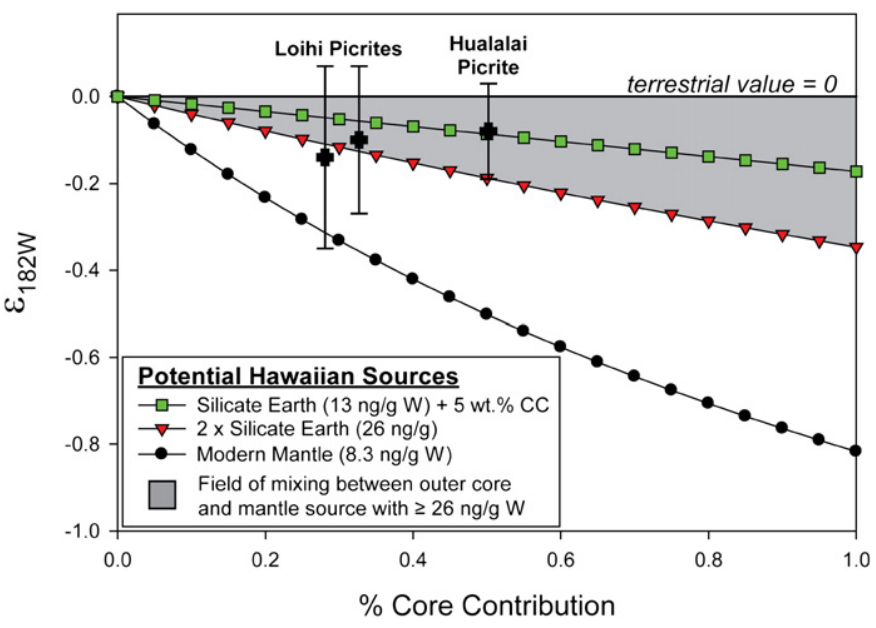

Fig. 5. Models of core-mantle exchange as detected by W isotopes (adapted from Brandon and Walker, 2005). Mixing between a model outer core (with $500 \mathrm{ng} / \mathrm{g} \mathrm{W}$, $\varepsilon_{182 \mathrm{~W}}=-2.1$ ) and several potential Hawaiian sources. A Hawaiian source equal to the mean modern mantle ( $8.3 \mathrm{ng} / \mathrm{g} \mathrm{W}$ ) cannot account for the lack of $\varepsilon_{182 \mathrm{~W}}$ depletions in the Hawaiian picrites suggested by Brandon et al. $(1998,1999)$ to record a core signature; this mixing trend mirrors the "preferred" core-mantle mixing curve of Scherstén et al. (2004). However, a more enriched source equal to two times the silicate Earth $(26 \mathrm{ng} / \mathrm{g} \mathrm{W}$ ), which is consistent with a deep mantle source defined below $\sim 1600 \mathrm{~km}$ depth, could account for the W isotopic signatures of the Hawaiian picrites. A Hawaiian source comprised of a silicate Earth component (13 ng/g W) plus 5 wt.\% continental crust (CC) could also explain the picrite data. Parameters for the continental crustal component were taken from Scherstén et al. (2004).

during the conditions of core formation, which is consistent with a deep $(>700 \mathrm{~km})$, potentially hydrous magma ocean early in Earth's history, according to high pressure-temperature metal/silicate partitioning experiments (Righter et al., 1997; Righter and Drake, 1999; Righter, 2003).

\subsection{Implications for the composition of the DMM and deep mantle}

The MORB source, often referred to as the depleted MORB mantle (or DMM), is generally thought to have formed as the result of an ancient depletion (i.e., continent formation). Although the source of MORB has been established to be compositionally heterogeneous (e.g., van Keken et al., 2002 and references therein), the relative depletion of this reservoir, as a whole, remains unsettled; this is largely due to difficulties in modeling the incompatible element budget of the DMM (Jagoutz et al., 1979; Hofmann, 1988, 2003).

Several studies have attempted to model the composition of the MORB source by analyzing the trace element chemistry of abyssal peridotites, calculating parent-daughter ratios of radiogenic isotopes, and using constant elemental concentration ratios. Assuming a chondritic original composition and a two-stage evolution of the continental crust, Salters and Stracke (2004) estimated an average DMM composition with $4.7 \pm 1.4(2 \sigma) \mathrm{ng} / \mathrm{g} \mathrm{U}$. This model represents a $\sim 4 \times$ depletion of the highly incompatible elements in the MORB source relative to the unfractionated silicate Earth, which is more depleted than the DMM composition of Boyet and Carlson (2006), which assumes a non-chondritic origin, but less depleted than the Workman and Hart (2005) model, which follows a gradual evolution of the continental crust. In order to further investigate the depletion of the DMM, we may also consider the incompatible trace element chemistry of global MORB in conjunction with simple partial melting dynamics. MORB from "typical" segments of mid-ocean ridges (those found far from known "plumes" or subduction zones) exhibit a mean composition of $16 \pm 13(2 \sigma) \mu \mathrm{g} / \mathrm{g}$ Ba (Su, 2002), a highly incompatible element comparable to U. Assuming $8-12 \%$ partial melting of the MORB source (e.g., Hofmann, 1988), this indicates that the DMM has approximately $1.6 \pm 1.3 \mu \mathrm{g} / \mathrm{g} \mathrm{Ba}$, verifying a factor of $\sim 4$ 
Table 3

Siderophile/lithophile element pairs

\begin{tabular}{llll}
\hline Element & Silicate earth $(\mu \mathrm{g} / \mathrm{g})^{\mathrm{a}}$ & Core $\mu \mathrm{g} / \mathrm{g})^{\mathrm{b}}$ & Core/SE enrichment factor \\
\hline $\mathrm{P}$ & 90 & 2000 & 22 \\
$\mathrm{Ti}$ & 1200 & 0 & 0.0 \\
$\mathrm{Mn}$ & 1045 & 300 & 0.3 \\
$\mathrm{Fe}$ & 62,600 & 855,000 & 14 \\
$\mathrm{Mo}$ & 0.05 & 5 & 100 \\
$\mathrm{Pd}$ & 0.004 & 3.1 & 775 \\
$\mathrm{Ba}$ & 6.6 & 0 & 0.0 \\
$\mathrm{Ce}$ & 1.68 & 0 & 0.0 \\
$\mathrm{Nd}$ & 1.25 & 0 & 0.0 \\
$\mathrm{Yb}$ & 0.44 & 0 & 0.0 \\
$\mathrm{~W}$ & 0.0131 & 0.504 & 38 \\
$\mathrm{Re}$ & 0.0003 & 0.23 & 767 \\
$\mathrm{~Pb}$ & 0.15 & 0.4 & 2.7 \\
$\mathrm{Fe} / \mathrm{Mn}$ & 60 & 2850 & $>10$ \\
$\mathrm{P} / \mathrm{Nd}$ & 72 & $>10^{5}$ & $>10^{3}$ \\
$\mathrm{~Pb} / \mathrm{Ce}$ & 0.089 & $>10^{3}$ & $>10^{4}$ \\
$\mathrm{Mo} / \mathrm{Ce}$ & 0.030 & $>10^{4}$ & $>10^{5}$ \\
$\mathrm{~W} / \mathrm{Ba}$ & 0.0020 & $>10^{3}$ & $>10^{5}$ \\
$\mathrm{Re} / \mathrm{Yb}$ & 0.00068 & $>10^{3}$ & $>10^{6}$ \\
$\mathrm{Pd} / \mathrm{Ti}$ & $3.3 \mathrm{E}-06$ & $>10^{4}$ & $>10^{9}$
\end{tabular}

${ }^{a}$ From McDonough and Sun (1995), excepting W.

b From McDonough (2003), excepting W.

depletion of highly incompatible elements in the MORB source compared to the bulk silicate Earth. According to the Salters and Stracke (2004) depletion model, the DMM is estimated to have $3.0 \pm$ $2.3(2 \sigma) \mathrm{ng} / \mathrm{g} \mathrm{W}$.

Constraining the mean abundance of $\mathrm{W}$ in the modern mantle and the composition of the MORB source region allows us to make some inferences as to the composition of the deep mantle, which has been shown to be geochemically distinct from the DMM (e.g., Hofmann, 1997). If we assume an upper and lower mantle separated by the $660 \mathrm{~km}$ discontinuity, this would necessitate $\sim 10 \mathrm{ng} / \mathrm{g} \mathrm{W}$ in the lower mantle, according to mass balance and a DMM composition of $3.0 \pm$ $2.3 \mathrm{ng} / \mathrm{g} \mathrm{W}$. However, it has been widely demonstrated that the $660 \mathrm{~km}$ discontinuity cannot serve as a chemical boundary, as a significant mass flux across this depth has been established by tomographic studies (e.g., Creager and Jordan, 1984; Davies and Richards, 1992; Grand et al., 1997; van der Hilst et al., 1997; Montelli et al., 2004; Nolet et al., 2006). Recently, seismological, geochemical and geophysical observations have identified a potentially deeper boundary layer between the upper and a heterogeneous deep mantle (e.g., Garnero, 2000 and references therein). A boundary layer at $\sim 1600 \mathrm{~km}$ depth, as preferred by Kellogg et al. (1999), would require an enriched deep mantle $\mathrm{W}$ abundance of $\sim 25 \mathrm{ng} / \mathrm{g}$, or roughly two times the W content of the unfractionated silicate Earth. Further, if we consider near-whole-mantle convection with the DMM constituting the entire mantle mass minus a 200-300 km thick boundary layer at the core-mantle boundary (which could serve as the early enriched reservoir invoked by Tolstikhin and Hofmann, 2005; Boyet and Carlson, 2005, 2006; Tolstikhin et al., 2006), this reservoir would contain $\sim 190 \mathrm{ng} / \mathrm{g} \mathrm{W}$.

As an additional consideration, we can examine the constancy of the $\mathrm{W} / \mathrm{Th}$ ratio in our basaltic dataset. Although the $\mathrm{W} / \mathrm{Th}$ ratio in the mantle is more variable than $\mathrm{W} / \mathrm{U}$ because it can be affected to some degree by partial melting (Fig. 3) and hydrothermal fluids (Noll et al., 1996), the mean $\mathrm{W} / \mathrm{Th}$ ratio of our oceanic basalts $(\mathrm{W} / \mathrm{Th}=0.19 \pm$ $0.15,2 \sigma)$ is comparable to the MORB and OIB dataset of Newsom et al. (1996; $\mathrm{W} / \mathrm{Th}=0.15 \pm 0.11,2 \sigma)$. As such, if the $\mathrm{W} / \mathrm{Th}$ ratio of the modern mantle is defined by our basaltic dataset, the mean mantle has a $\mathrm{Th} / \mathrm{U}$ ratio of $3.3 \pm 1.4(2 \sigma)$, which is higher than estimates of the MORB source (e.g., 2.5; Turcotte et al., 2001) but lower than the preferred value for the silicate Earth (e.g., 3.9; McDonough and Sun, 1995) and chondritic meteorites (e.g., 3.8; Rocholl and Jochum, 1993).

\subsection{Detecting core-mantle interactions through $W$ isotopes}

Excesses of isotope ${ }^{182} \mathrm{~W}$ are produced via beta-decay of the nowextinct ${ }^{182} \mathrm{Hf}$ nuclide, which has a half-life of $8.90 \pm 0.09 \mathrm{Ma}$ (Vockenhuber et al., 2004). Both Hf and W are refractory elements, but differ in their geochemical affinities; under the reducing conditions of core formation, $\mathrm{Hf}$ acts as a lithophile element, and thus prefers to remain in silicate phases, whereas $\mathrm{W}$ behaves as a moderately siderophile element. Consequently, a metallic core will have a $\mathrm{Hf} / \mathrm{W}$ ratio of $\sim 0$ and will therefore retain its original $\mathrm{W}$ isotopic composition. Because of the short-lived nature of this radiogenic system, any existing $\mathrm{W}$ isotopic heterogeneities within the Earth must have been inherited during the first $\sim 50 \mathrm{Ma}$ of solar system history, the functional lifetime of ${ }^{182} \mathrm{Hf}$.

Studies of terrestrial samples and chondritic meteorites have established that the core of the Earth must be depleted by $\sim 2$ parts per 10,000 (or $2 \varepsilon$-unit) in ${ }^{182} \mathrm{~W}$ relative to the silicate Earth (Kleine et al., 2002; Schoenberg et al., 2002, Yin et al., 2002). Because the concentration of $\mathrm{W}$ is significantly greater in the core compared to the mantle, small depletions in ${ }^{182} \mathrm{~W}$ (relative to the silicate Earth) in "plume-derived" magmas that originate near the base of the mantle may result from core-mantle interactions. However, initial investigations into the $\mathrm{W}$ isotopic composition of several Hawaiian picrites that may record a core component in their source (on the basis of resolvable enrichments in ${ }^{186}$ Os- ${ }^{187}$ Os; Brandon et al., 1998, 1999) failed to resolve these rocks from terrestrial standards (Scherstén et al., 2004).

The $\mathrm{W}$ content of the three Hawaiian picrites measured by Scherstén et al. (2004) range from $113 \mathrm{ng} / \mathrm{g}(\mathrm{H}-11)$ to $313 \mathrm{ng} / \mathrm{g}$ (Lo02-04), as determined by this study. Assuming 5-15\% partial melting, this indicates a Hawaiian source with anywhere from 5 to $45 \mathrm{ng} / \mathrm{g} \mathrm{W}$. Assuming a Hawaiian source with a concentration of $26 \mathrm{ng} / \mathrm{g} \mathrm{W}$, or approximately two times the $\mathrm{W}$ content of the undifferentiated silicate Earth (13 ng/g W), the three Hawaiian picrites may be accounted for by a two end-member mixing scenario between the outer core and an enriched deep mantle reservoir (Fig. 5). Such an

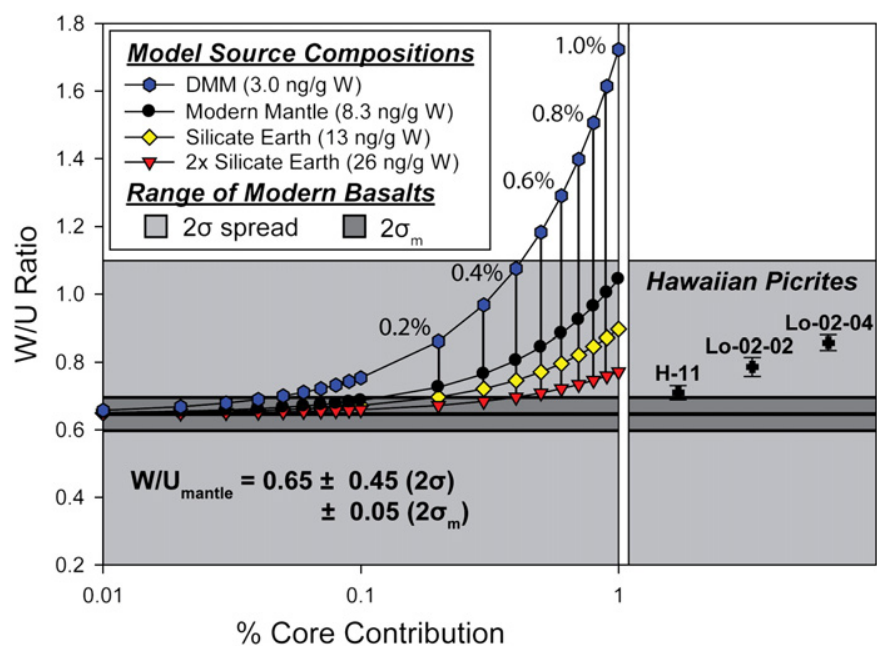

Fig. 6. Two end-member mixing between the outer core and model mantle sources. The light gray field represents the $2 \sigma$ scatter seen in our mantle-derived samples; the dark gray region represents the $2 \sigma_{\mathrm{m}}$. The overall range of $\mathrm{W} / \mathrm{U}$ seen in modern oceanic basalts makes assessing core-mantle exchange difficult for enriched sources and low degrees of core contribution. As seen in the figure, even up to $1 \mathrm{wt}$ \% of core material added to a mantle source with $\geq 8.3 \mathrm{ng} / \mathrm{g} \mathrm{W}$ would not result in an enriched $\mathrm{W} / \mathrm{U}$ outside of the spread seen in basaltic sources. However, a more depleted source, such as a source equal to the Depleted MORB Mantle (i.e., DMM), would allow for the detection of $<1.0 \mathrm{wt}$ \% core contribution. Data for the Hawaiian picrites examined by Brandon et al. $(1998,1999)$ and Scherstén et al. (2004) lay above the mean and $2 \sigma_{\mathrm{m}}$ spread, but easily reside within the scatter seen in our basaltic samples. This does not preclude a core component added to the source of these samples, though, as the source of these picrites likely has $>3.0 \mathrm{ng} / \mathrm{g} \mathrm{W}$. 
enriched Hawaiian source is consistent with a lower mantle source defined below $\sim 1600 \mathrm{~km}$ depth, as originally suggested by Kellogg et al. (1999), and may result from pyroxenitic melting in the Hawaiian source region (Sobolev et al., 2005, 2007). Additionally, as Brandon and Walker (2005) observed, even modest contamination by a crustal component could overprint any $\mathrm{W}$ core signature recorded in these rocks while having a negligible effect on their Os isotopic ratios. This is illustrated in the model source consisting of a silicate Earth component, with $13 \mathrm{ng} / \mathrm{g} \mathrm{W}$, mixed with 5 wt.\% continental crust in Fig. 5. Although higher-precision measurements of the $\mathrm{W}$ isotopic compositions of these rocks might provide some clarity to these issues, an additional window into detecting core-mantle exchange may lie with the terrestrial $\mathrm{W} / \mathrm{U}$ ratio.

\subsection{Detecting core-mantle interactions through $W$ concentration ratios}

Whereas isotopic ratios can serve as geochemical tracers of either the physical admixture of or diffusive isotopic exchange between the core and mantle (Puchtel and Humayun, 2000), concentration ratios can only act as a proxy of the physical admixture of these two reservoirs, assuming the core and silicate Earth (including both the modern mantle and continental crustal reservoirs) each have a unique and constant value. Because core formation has depleted the silicate Earth in siderophile elements relative to the lithophile elements, siderophile-to-lithophile ratios provide the most sensitive type of tracers because such ratios are extremely high in the core and correspondingly low in the silicate Earth (Table 3). For example, Fe/Mn ratios, which have been implicated in the identification of a core signature in modern Hawaiian lavas (Humayun et al., 2004), are enriched in the core relative to the mantle, but only by a factor of $>10$. On the other hand, the $\mathrm{W} / \mathrm{U}$ of the core is enriched by a factor $>10^{5}$ relative to the modern mantle. As a result, $\mathrm{W} / \mathrm{U}$ is potentially threetimes more sensitive than $\mathrm{Fe} / \mathrm{Mn}$ to a two end-member mixing scenario between the mantle and $1 \mathrm{wt} . \%$ of core material, considering the reservoir compositions listed in Table 3.

Since the core contains approximately 95\% of the bulk Earth's W budget and essentially no U (McDonough, 2003), it can be confidently established that the core has a $W / U \gg 1000$. A two end-member mixing curve between a mantle reservoir with $\geq 8.3 \mathrm{ng} / \mathrm{g} \mathrm{W}$ and $\mathrm{W} /$ $\mathrm{U}=0.65 \pm 0.45(2 \sigma)$ and an outer core reservoir with $\sim 500 \mathrm{ng} / \mathrm{g} \mathrm{W}$ and $\mathrm{W} / \mathrm{U}=1000$ (as a lower limit), however, indicates that the physical entrainment of up to $1 \mathrm{wt}$.\% of outer core materials would fail to result in a resolvable increase in $\mathrm{W} / \mathrm{U}$; a more depleted source composition (e.g., DMM with $3.0 \mathrm{ng} / \mathrm{g} \mathrm{W}$ ) or a higher degree of core contamination is required for core-mantle exchange to be identified through the use of this proxy (Fig. 6). An analysis of the three Hawaiian picrites examined by Brandon et al. $(1998,1999)$ and Scherstén et al. (2004) illustrates these limitations. Although all three of the picrites reside within the variability seen in our sample suite, this does not preclude a core component added to the source of these rocks. Brandon et al $(1998,1999)$ suggest that these three picrites carry only $0.3-0.5 \%$ core contribution; this amount of mass exchange is permitted with our mixing models assuming a Hawaiian source with an enriched mantle composition. Thus, although $\mathrm{W} / \mathrm{U}$ serves as a potentially sensitive geochemical tracer of physical admixture between outer core and deep mantle materials, the variability seen in modern basaltic samples makes it difficult to unambiguously detect core contributions in materials derived from enriched source regions.

\section{Conclusions}

Modern oceanic basalts, including both E-type and N-type MORB, intraplate and convergent margin volcanics, reveal that $\mathrm{U}$ behaves analogously to $\mathrm{W}$ during silicate differentiation. The average $\mathrm{W} / \mathrm{U}$ ratio of these samples $(0.65 \pm 0.45,2 \sigma, n=86)$ is representative of both the modern mantle and bulk silicate Earth, and suggests a bulk silicate
Earth composition of $13 \pm 10 \mathrm{ng} / \mathrm{g} \mathrm{W}$ and a mean modern mantle composition of $8.3 \pm 7.1 \mathrm{ng} / \mathrm{g} \mathrm{W}$. According to the model DMM composition of Salters and Stracke (2004), the MORB source likely contains $3.0 \pm 2.3 \mathrm{ng} / \mathrm{g} \mathrm{W}$. Assuming a stratified mantle at $\sim 1600 \mathrm{~km}$ depth, this indicates a deep mantle with $\sim 25 \mathrm{ng} / \mathrm{g} \mathrm{W}$. Alternatively, considering near-whole-mantle convection with only a $\sim 250 \mathrm{~km}$ thick $D^{\prime \prime}$ layer at the core-mantle boundary, this reservoir must be enriched up to $190 \mathrm{ng} / \mathrm{g} \mathrm{W}$.

In theory, both $\mathrm{W}$ isotopes and $\mathrm{W} / \mathrm{U}$ ratios can be used to identify core-mantle exchange. However, as with most geochemical tracers of internal processes within the Earth, these proxies are dependent on the source composition of the sampled materials. Three Hawaiian picrites initially examined by Brandon et al. $(1998,1999)$ have been suggested to record a core signature according to their ${ }^{186} \mathrm{Os}-{ }^{187} \mathrm{Os}$ isotopic compositions. However, no discernable core signature is seen via $\mathrm{W}$ isotopes or $\mathrm{W} / \mathrm{U}$ ratios. This suggests either (1) the lack of a core component in the source of these rocks, (2) crustal contamination of the samples, or (3) a source with at least $26 \mathrm{ng} / \mathrm{g} \mathrm{W}$, which is consistent with a deep mantle source defined below $1600 \mathrm{~km}$. Accordingly, until further constraints can be placed on the source region of these samples, the issue of core-mantle exchange has yet to be resolved.

\section{Acknowledgements}

We would like to thank the Division of Petrology and Volcanology, Department of Mineral Sciences, Smithsonian Institute, especially Leslie Hale, Sorena Sorenson and the late Jim Luhr, for providing us with so many precious samples to conduct this study. We also appreciate the sample donations generously afforded by Michael Garcia, Thomas Ireland, Emily Klein, Charles Langmuir, Gaby Loock, Yaoling Niu, Marc Norman, and Michael Perfit. This study could not have been conducted without the analytical prowess of Richard Ash, who helped us develop the analytical protocol for our LA-ICP-MS method, or the helpful reviews of Rick Carlson, James Day, John Lassiter, Adam Mansur, Carsten Münker, Sune Nielsen, and Richard Walker. Special thanks go to Thorsten Kleine and Tetsuya Yokoyama for their assistance with developing our $\mathrm{W}$ isotope dilution procedures. This study was funded by NSF grant \# 0337621 and \#0739006.

\section{Appendix A. Supplementary data}

Supplementary data associated with this article can be found, in the online version, at doi:10.1016/j.epsl.2008.05.031.

\section{References}

Bevington, P.R., Robinson, D.K., 2003. Data Reduction and Error Analysis for the Physical Sciences. McGraw-Hill, New York, NY.

Boyet, M., Carlson, R.W., 2005. ${ }^{142} \mathrm{Nd}$ evidence for early ( $>4.53 \mathrm{Ga}$ ) global differentiation of the silicate Earth. Science 309, 576-581.

Boyet, M., Carlson, R.W., 2006. A new geochemical model for the Earth's mantle inferred from ${ }^{146} \mathrm{Sm}-{ }^{142} \mathrm{Nd}$ systematics. Earth Planet. Sci. Lett. 250, 254-268.

Brandon, A.D., Walker, R.J., 2005. The debate over core-mantle interaction. Earth Planet. Sci. Lett. 232, 211-225.

Brandon, A.D., Walker, R.J., Morgan, J.W., Norman, M.D., Prichard, H.M., 1998. Coupled ${ }^{186}$ Os and ${ }^{187}$ Os evidence for core-mantle interaction. Science 280, 1570-1573.

Brandon, A.D., Norman, M.D., Walker, R.J., Morgan, J.W., 1999. ${ }^{186}$ Os $-{ }^{187}$ Os systematics of Hawaiian picrites. Earth Planet. Sci. Lett. 174, 25-42.

Creager, K.C., Jordan, T.H., 1984. Slab penetration into the lower mantle. J. Geophys. Res. 89, 3031-3049.

Crisp, J.A., 1984. Rates of magma emplacement and volcanic output. J. Volc. Geotherm. Res. 20, 177-211.

Davies, G.F. Richards, M.A., 1992. Mantle convection. J. Geol. 100, 151-206.

Eggins, S.M., Shelley, J.M.G., 2002. Compositional heterogeneity in NIST SRM 610-617 glasses. Geostd. News. 26, 269-286.

Eggins, S.M., Rudnick, R.L., McDonough, W.F., 1998. The composition of peridotites and their minerals: a laser-ablation ICP-MS study. Earth Planet. Sci. Lett. 154, 53-71.

Garnero, E.J., 2000. Heterogeneity of the lowermost mantle. Annu. Rev. Earth Planet. Sci. 28, 509-537.

Grand, S., van der Hilst, R.D., Widiyantoro, S., 1997. Global seismic tomography: a snapshot of convection in the Earth. GSA Today 7,1-7. 
Hofmann, A.W., 1988. Chemical differentiation of the Earth - the relationship between mantle, continental-crust, and oceanic-crust. Earth Planet. Sci. Lett. 90, 297-314.

Hofmann, A.W., 1997. Mantle geochemistry: the message from oceanic volcanism. Nature 385, 219-229.

Hofmann, A.W., 2003. Sampling mantle heterogeneity through oceanic basalts: isotopes and trace elements, The Mantle and Core (ed. R.W. Carlson) Vol. 2 Treatise on Geochemistry (eds. H.D. Holland and K.K Turekian). Elsevier-Pergamon, Oxford, pp. 61-101.

Hofmann, A.W., Jochum, K.P., Seufert, M., White, W.M., 1986. Nb and Pb in oceanic basalts - new constraints on mantle evolution. Earth Planet. Sci. Lett. 79, 33-45.

Humayun, M., Qin, L.P., Norman, M.D., 2004. Geochemical evidence for excess iron in the mantle beneath Hawaii. Science 306, 91-94.

Jacobsen, S.B., 2005. The Hf-W isotopic system and the origin of the earth and moon. Annu. Rev. Earth Planet. Sci. 33, 531-570.

Jagoutz, E., Palme, H., Baddenhausen, H., Blum, K., Cendales, M., Dreibus, G., Spettel, B., Lorenz, V., Wanke, H., 1979. The abundances of major, minor and trace elements in the earth's mantle as derived from primitive ultramafic nodules. 10th Proceedings of Lunar and Planetary Sciences, pp. 2031-2050.

Jochum, K.P., McDonough, W.F., Palme, H., Spettel, B., 1989. Compositional constraints on the continental lithospheric mantle from trace elements in spinel peridotite xenoliths. Nature 340, 548-550.

Jochum, K.P., Stoll, B., Herwig, K., Willbold, M., Hofmann, A.W., Amini, M., Aarburg, S., Abouchami, W., Hellebrand, E., Mocek, B., Raczek, I., Stracke, A., Alard, O., Bouman, C., Becker, S., Ducking, M., Bratz, H., Klemd, R., de Bruin, D., Canil, D., Cornell, D., de Hoog, C.J., Dalpe, C., Danyushevsky, L., Eisenhauer, A., Premo, W.R., Sun, W.D.D., Tiepolo, M., Vannucci, R., Vennemann, T., Wayne, D., Woodhead, J.D., 2006. MPIDING reference glasses for in situ microanalysis: new reference values for element concentrations and isotope ratios. Geochem. Geophys. Geosyst. 7, 1-44.

Jochum, K.P., Willbold, M., Raczek, I., Stoll, B., Herwig, K., 2005. Chemical characterisation of the USGS reference glasses GSA-1G, GSC-1G, GSD-1G, GSE-1G, BCR-2G, BHVO-2G and BIR-1G using EPMA, ID-TIMS, ID-ICP-MS and LA-ICP-MS. Geostd. Geoanal. Res. 29, 285-302.

Jochum, K.P., Stoll, B., Herwig, K., Willbold, M., 2007. Validation of LA-ICP-MS trace element analysis of geological glasses using a new solid-state $193 \mathrm{~nm} \mathrm{Nd}$ :YAG laser and matrix-matched calibration. J. Anal. Atom. Spec. 22, 112-121.

Kellogg, L.H., Hager, B.H., van der Hilst, R.D., 1999. Compositional stratification in the deep mantle. Science 283, 1881-1884

Kleine, T., Munker, C., Mezger, K., Palme, H., 2002. Rapid accretion and early core formation on asteroids and the terrestrial planets from $\mathrm{Hf}-\mathrm{W}$ chronometry. Nature 418, 952-955.

Kleine, T., Mezger, K., Palme, H., Munker, C., 2004a. The W isotope evolution of the bulk silicate Earth: constraints on the timing and mechanisms of core formation and accretion. Earth Planet. Sci. Lett. 228, 109-123.

Kleine, T., Mezger, K., Munker, C., Palme, H., Bischoff, A., 2004b. ${ }^{182} \mathrm{Hf}-{ }^{182} \mathrm{~W}$ isotope systematics of chondrites, eucrites, and martian meteorites: chronology of core formation and early mantle differentiation in Vesta and Mars. Geochim. Cosmochim. Acta 68, 2935-2946.

Kleine, T., Mezger, K., Palme, H., Scherer, E., Munker, C., 2005a. Early core formation in asteroids and late accretion of chondrite parent bodies: evidence from ${ }^{182} \mathrm{Hf}-{ }^{182} \mathrm{~W}$ in CAIs, metalrich chondrites, and iron meteorites. Geochim. Cosmochim. Acta 69, 5805-5818.

Kleine, T., Palme, H., Mezger, M., Halliday, A.N., 2005b. Hf-W chronometry of lunar metals and the age and early differentiation of the Moon. Science 310, 1671-1674.

Lassiter, J.C., 2004. Role of recycled oceanic crust in the potassium and argon budget of the Earth: toward a resolution of the "missing argon" problem. Geochem. Geophys. Geosyst. 5, 1-16.

Loock, G., McDonough, W.F., Goldstein, S.L., Hofmann, A.W., 1990. Isotopic compositions of volcanic glasses from the Lau Basin. Marine Mining 9, 235-245.

Lyubetskaya, T., Korenaga, J., 2007. Chemical composition of Earth's primitive mantle and its variance: 1. Method and results. J. Geophys. Res. 112, B03212. doi:10.1029/ 2005JB004224.

McDonough, W.F., 1991. Partial melting of subducted oceanic crust and isolation of its residual eclogitic lithology. Phil. Trans. Roy. Soc. Lond. 335, 407-418.

McDonough, W.F., 2003. Compositional model for the Earth's core, The Mantle and Core (ed. R.W. Carlson) Vol. 2 Treatise on Geochemistry (eds. H.D. Holland and K.K Turekian). Elsevier-Pergamon, Oxford, pp. 547-568.

McDonough, W.F., Sun, S.S., 1995. The composition of the Earth. Chem. Geol. 120, 223-253.

Montelli, R., Nolet, G., Dahlen, F.A., Masters, G., Engdahl, E.R., Hung, S.H., 2004. Finitefrequency tomography reveals a variety of plumes in the mantle. Science 303, 338-343.

Newsom, H.E., 1990. Accretion and core formation in the Earth: evidence from siderophile elements. In: Newsom, H.E., Jones, J.H. (Eds.), Origin of Earth. Oxford Press, pp. 273-288.

Newsom, H.E., Palme, H., 1984. The depletion of siderophile elements in the Earth's mantle new evidence from molybdenum and tungsten. Earth Planet. Sci. Lett. 69, 354-364.

Newsom, H.E., White, W.M., Jochum, K.P., Hofmann, A.W., 1986. Siderophile and chalcophile element abundances in oceanic basalts, Pb-isotope evolution and growth of the Earth's core. Earth Planet. Sci. Lett. 80, 299-313.

Newsom, H.E., Sims, K.W.W., Noll, P.D., Jaeger, W.L., Maehr, S.A., Beserra, T.B., 1996. The depletion of tungsten in the bulk silicate Earth: constraints on core formation. Geochim. Cosmochim. Acta 60, 1155-1169.

Niu, Y.L., Batiza, R., 1997. Trace element evidence from seamounts for recycled oceanic crust in the eastern Pacific mantle. Earth Planet. Sci. Lett. 148, 471-483.

Nolet, G., Karato, S.I., Montelli, R., 2006. Plume fluxes from seismic tomography. Earth Planet. Sci. Lett. 248, 685-699.

Noll, P.D., Newsom, H.E., Leeman, W.P., Ryan, J.G., 1996. The role of hydrothermal fluids in the production of subduction zone magmas: evidence from siderophile and chalcophile trace elements and boron. Geochim. Cosmochim. Acta 60, 587-611.
Norman, M.D., Griffin, W.L., Pearson, N.J., Garcia, M.O., O'Reilly, S.Y., 1998. Quantitative analysis of trace element abundances in glasses and minerals: a comparison of laser ablation inductively coupled plasma mass spectrometry, solution inductively coupled plasma mass spectrometry, proton microprobe and electron microprobe data. J. Analyt. Atom. Spec. 13, 477-482.

Palme, H., Rammensee, W., 1981. The significance of $\mathrm{W}$ in planetary differentiation processes: evidence from new data on eucrites. 12th Lunar and Planetary Sciences Conference, pp. 949-964.

Palme, H. and O'Neill, H.S.C., 2003. Cosmochemical estimates of mantle composition, The Mantle and Core (ed. R.W. Carlson) Vol. 2 Treatise on Geochemistry (eds. H.D. Holland and K.K Turekian). Elsevier-Pergamon, Oxford, pp. 1-38.

Pearce, N.J.G., Perkins, W.T., Westgate, J.A., Gorton, M.P., Jackson, S.E., Neal, C.R., Chenery, S.P., 1997. A compilation of new and published major and trace element data for NIST SRM 610 and NIST SRM 612 glass reference materials. Geostd. News. 21, 115-144.

Puchtel, I., Humayun, M., 2000. Platinum group elements in Kostomuksha komatiites and basalts: Implications for oceanic crust recycling and core-mantle interaction. Geochim. Cosmochim. Acta 64, 4227-4242.

Righter, K., 2003. Metal-silicate partitioning of siderophile elements and core formation in the early Earth. Annu. Rev. Earth Planet. Sci. 31, 135-174.

Righter, K., Drake, M.J., 1999. Effect of water on metal-silicate partitioning of siderophile elements: a high pressure and temperature terrestrial magma ocean and core formation. Earth Planet. Sci. Lett. 171, 383-399.

Righter, K., Shearer, C.K., 2003. Magmatic fractionation of Hf and W: constraints on the timing of core formation and differentiation in the Moon and Mars. Geochim. Cosmochim. Acta 67, 2497-2507.

Righter, K., Drake, M.J., Yaxley, G., 1997. Prediction of siderophile element metal-silicate partition coefficients to $20 \mathrm{GPa}$ and 2800 degrees C: the effects of pressure, temperature, oxygen fugacity, and silicate and metallic melt compositions. Phys. Earth Planet. Int. 100, 115-134.

Rocholl, A., Jochum, K.P., 1993. Th, U and other trace-elements in carbonaceous chondrites - implications for the terrestrial and solar-system Th/U ratios. Earth Planet. Sci. Lett. 117, 265-278.

Rudnick, R.L. and Gao, S., 2003. Composition of the continental crust, The Crust (ed. R.L. Rudnick) Vol. 3 Treatise on Geochemistry (eds. H.D. Holland and K.K Turekian) Elsevier-Pergamon, Oxford, pp. 1-64.

Salters, V.J.M., Stracke, A., 2004. Composition of the depleted mantle. Geochem. Geophys. Geosyst. 5, 1-27.

Scherstén, A., Elliott, T., Hawkesworth, C., Norman, M., 2004. Tungsten isotope evidence that mantle plumes contain no contribution from the Earth's core. Nature 427, 234-237.

Schoenberg, R., Kamber, B.S., Collerson, K.D., Eugster, O., 2002. New W-isotope evidence for rapid terrestrial accretion and very early core formation. Geochim. Cosmochim. Acta $66,3151-3160$

Sims, K.W., Newsom, H.E., Gladney, E.S., 1990. Chemical fractionation during formation of the Earth's core and continental crust: clues from As, Sb, W, and Mo. In: Newsom, H.E., Jones, J.H. (Eds.), Origin of Earth. Oxford Press, pp. 291-317.

Sims, K.W.W., DePaolo, D.J., 1997. Inferences about mantle magma sources from incompatible element concentration ratios in oceanic basalts. Geochim. Cosmochim. Acta 61, 765-784.

Sobolev, A.V., Hofmann, A.W., Kuzmin, D.V., Yaxley, G.M., Arndt, N.T., Chung, S.L., Danyushevsky, L.V., Elliott, T., Frey, F.A., Garcia, M.O., Gurenko, A.A., Kamenetsky, V.S., Kerr, A.C., Krivolutskaya, N.A., Matvienkov, V.V., Nikogosian, I.K., Rocholl, A., Sigurdsson, I.A., Sushchevskaya, N.M., Teklay, M., 2007. The amount of recycled crust in sources of mantle-derived melts. Science 316, 412-417.

Sobolev, A.V., Hofmann, A.W., Sobolev, S.V., Nikogosian, I.K., 2005. An olivine-free mantle source of Hawaiian shield basalts. Nature 434, 590-597.

$\mathrm{Su}$, Y.J., 2002. Mid-ocean ridge basalt trace element systematics: constraints from database management, ICP-MS analyses, global data compilation, and petrologic modeling, Ph.D. Columbia University, pp. 457.

Sun, S.S., 1982. Chemical composition and origin of the earth's primitive mantle. Geochim. Cosmochim. Acta 46, 179-192.

Sun, S.-S., McDonough, W.F., 1989. Chemical and isotopic systematics of oceanic basalts: implications for mantle composition and processes. Magmatism in the Ocean Basins, vol. 42. Geological Society Special Publication.

Taylor, S.R., McLennan, S.M., 1985. The Continental Crust: Its Composition and Evolution. Blackwell, Oxford.

Tolstikhin, I., Hofmann, A.W., 2005. Early crust on top of the Earth's core. Phys. Earth Planet. Int. 148, 109-130.

Tolstikhin, I.N., Kramers, J.D., Hofmann, A.W., 2006. A chemical Earth model with whole mantle convection: the importance of a core-mantle boundary layer $\left(D^{\prime \prime}\right)$ and its early formation. Chem. Geol. 226, 79-99.

Turcotte, D.L., Paul, D., White, W.M., 2001. Thorium-uranium systematics require layered mantle convection. J. Geophys. Res. 106, 4265-4276.

van der Hilst, R.D., Widiyantoro, S., Engdahl, E.R., 1997. Evidence for deep mantle circulation from global tomography. Nature 386, 578-584.

van Keken, P.E., Hauri, E.H., Ballentine, C.J., 2002. Mantle mixing: the generation, preservation, and destruction of chemical heterogeneity. Annu. Rev. Earth Planet. Sci. 30, 493-525.

Vockenhuber, C., Oberli, F., Bichler, M., Ahmad, I., Quitte, G., Meier, M., Halliday, A.N., Lee, D. C., Kutschera, W., Steier, P., Gehrke, R.J., Helmer, R.G., 2004. New half-life measurement of ${ }^{182} \mathrm{Hf}$ : improved chronometer for the early solar system. Phys. Rev. Lett. 93,1-4.

Workman, R.K., Hart, S.R., 2005. Major and trace element composition of the depleted MORB mantle (DMM). Earth Planet. Sci. Lett. 231, 53-72.

Yin, Q.Z., Jacobsen, S.B., Yamashita, K., Blichert-Toft, J., Telouk, P., Albaréde, F., 2002. A short timescale for terrestrial planet formation from Hf-W chronometry of meteorites. Nature 418, 949-952. 